\title{
IMPACT OF AN L5 MAGNETOGRAPH ON NONPOTENTIAL SOLAR GLOBAL MAGNETIC FIELD
} MODELING

\author{
Duncan H. Mackay ${ }^{1}$, Anthony R. Yeates $^{2}$, and Francois-Xavier BocQuet ${ }^{3}$ \\ ${ }^{1}$ School of Mathematics and Statistics, University of St Andrews, North Haugh, St Andrews, Fife, Scotland, KY16 9SS, UK; dhm@st-andrews.ac.uk \\ ${ }^{2}$ Department of Mathematical Sciences, Durham University, Science Laboratories, South Road, Durham, DH1 3LE, UK \\ ${ }^{3}$ Met Office, FitzRoy Road, Exeter, EX1 3PB, UK \\ Received 2016 March 16; revised 2016 May 13; accepted 2016 May 14; published 2016 July 12
}

\begin{abstract}
We present the first theoretical study to consider what improvement could be obtained in global nonpotential modeling of the solar corona if magnetograph data were available from the L5 Lagrange point, in addition to from the direction of Earth. To consider this, we first carry out a "reference Sun" simulation over two solar cycles. An important property of this simulation is that random bipole emergences are allowed across the entire solar surface at any given time (such as can occur on the Sun). Next, we construct two "limited data" simulations, where bipoles are only included when they could be seen from (i) an Earth-based magnetograph and (ii) either Earth- or L5-based magnetographs. The improvement in reproducing the reference Sun simulation when an L5 view is available is quantified through considering global quantities in the limited data simulations. These include surface and polar flux, total magnetic energy, volume electric current, open flux, and the number of flux ropes. Results show that when an L5 observational viewpoint is included, the accuracy of the global quantities in the limited data simulations can increase by $26 \%-40 \%$. This clearly shows that a magnetograph at the L5 point could significantly increase the accuracy of global nonpotential modeling and with this the accuracy of future space weather forecasts.
\end{abstract}

Key words: Sun: activity - Sun: corona - Sun: magnetic fields

\section{INTRODUCTION}

The phrase "space weather" describes the collective effect that the Sun has on the near-Earth environment. A wide variety of phenomena on the Sun, both eruptive and noneruptive, can lead to space weather events. Such phenomena include solar flares (Benz 2008), coronal mass ejections (Forbes et al. 2006), solar filaments (Labrosse et al. 2010; Mackay et al. 2010), the Sun's open magnetic flux (Balogh et al. 1995), and the solar wind (Hollweg 2008). Space weather events vary widely in severity. At the least disruptive end, as the Earth orbits the Sun there is a continual interaction between the Earth's magnetosphere and the Sun's open flux and solar wind. Such interaction results in the ambient space weather encountered at Earth. In contrast, eruptive phenomena on the Sun can lead to sporadic but severe space weather events. During these, the Earth's magnetosphere experiences significant disruption. This disruption can have adverse effects on modern technological systems, such as the shorting of power grids, disruption in communications, and loss of GPS systems. In the worst cases severe space weather events may lead to the destruction of satellites or, in principle, to loss of life in manned space missions. Due to the recognized international importance of space weather, both the $\mathrm{US}^{4}$ and $\mathrm{UK}^{5}$ issue $24 / 7$ space weather predictions and warnings. While many pieces of information are required for effective predictions, including both observational and theoretical components, two elements are fundamental to effective predictions. The first is accurate observations of plasma in the Sun's atmosphere and heliosphere. The second is knowledge of the nonpotential state of the Sun's magnetic field both at the photosphere and in the corona. The latter requires some form of theoretical modeling.

\footnotetext{
4 http://www.swpc.noaa.gov/

5 http://www.metoffice.gov.uk/publicsector/emergencies/space-weather
}

One aspect that limits our understanding of the origin and evolution of space weather phenomena on the Sun is our limited field of view (FOV) of the Sun. Most current observations are taken from the Sun-Earth line, except those from the NASA/STEREO satellites. This limited FOV means that we have limited information on solar phenomena that are rotating toward us until 5-7 days before they reach central meridian on the Earth-Sun line. This restricts long-term predictive capabilities. In order to improve predictions, it is proposed to place a satellite at the L5 Lagrange point, which lies $60^{\circ}$ behind the orbit of the Earth. This stable orbit point provides an additional 5-6 days early warning of possible space weather producing phenomena as they rotate toward the Earth. A wide variety of instruments are proposed for the L5 mission and include both remote sensing (coronagraph, heliospheric imager, EUV imager, and line-of-sight [LOS] magnetograph) and in situ capabilities (for measuring particles and fields). The focus of this paper is on one of the possible remote sensing instruments: a normal component magnetograph. The goal is to quantify how magnetograph observations of the Sun's photospheric magnetic field from an L5 mission could improve the accuracy of global nonpotential models.

Over the past $20 \mathrm{yr}$, a wide range of global nonpotential models in spherical coordinates have been developed to model the coronal magnetic field of the Sun based on input of photospheric magnetograms. For a full review see Mackay \& Yeates (2012) and references therein. One difficulty that arises in using these models is that we can only observe one side of the Sun at a single time, but need data for all longitudes. Normally this problem is addressed either by compiling a time series of full-disk observations into synoptic magnetograms or by assimilating individual active regions into a time-dependent surface flux transport model (Sheeley 2005). One important benefit of an L5 magnetograph would be to provide an additional $60^{\circ}$ of accurate magnetic field measurements to 
incorporate into the photospheric boundary condition magnetograms used by the models. Currently, global nonpotential models use a variety of approximations from nonlinear forcefree fields (Mackay \& van Ballegooijen 2006; Wiegelmann 2007; Yeates et al. 2008; Contopoulos et al. 2011) to magnetohydrostatic models (Wiegelmann et al. 2007) and finally full MHD simulations (Riley et al. 2006; DeVore \& Antiochos 2008; Lionello et al. 2009; Downs et al. 2010; Feng et al. 2012).

In the present paper we will consider what effect an LOS magnetograph at the L5 point would have on the global nonpotential model of van Ballegooijen et al. (2000) and Mackay \& van Ballegooijen (2006). The model follows the long-term evolution of the coronal magnetic field over periods of months to years. It describes the buildup of free magnetic energy and electric currents in the corona by coupling together two distinct models. The first is a data-driven surface flux transport model (Yeates et al. 2007). This uses observations of newly emerging magnetic bipoles, along with well-observed global motions, to produce a continuous evolution of the observed photospheric magnetic flux over long periods of time. Coupled to this is a quasi-static coronal evolution model (Mackay \& van Ballegooijen 2006; Yeates et al. 2008), which evolves the coronal magnetic field through a sequence of nonlinear force-free states in response to the observed photospheric evolution and flux emergence. As Yeates (2014) has shown, the model may be used instead of MHD simulations to describe the corona over solar cycle timescales. Previously, the model has been successfully applied to consider the hemispheric pattern of filaments (Yeates et al. 2008; Yeates \& Mackay 2012), the variation in the Sun's open magnetic flux (Yeates et al. 2010), the formation of magnetic flux ropes (Yeates \& Mackay 2009), and their subsequent loss of equilibrium into a coronal mass ejection (CME; Pagano et al. 2013, 2014). Due to its previous success in describing nonpotential coronal phenomena, many of which are related to space weather, it is reasonable to use the model in a speculative way to determine how a magnetograph at the L5 point would improve the model's accuracy. In addition to improving global nonpotential models, magnetograph data from an L5 mission may also improve other types of predictive models with space weather applications. In particular, the increased magnetograph FOV should increase the accuracy in models for calculating the solar spectral irradiance (Fontenla et al. 2009a, 2009b) or EUV/F10.7 flux (Henney et al. 2015). These models are important for space weather as they can be coupled with both ionospheric and thermospheric models. While we focus on our own global nonpotential model, we encourage similar studies with other space-weather-related models.

In order to quantify what impact a magnetograph at the L5 point would have on the accuracy of our global nonpotential model, we carry out three simulations. The first simulation, which we name the "reference Sun" simulation, is a whole-Sun simulation where the emergence of bipoles is known at all longitudes throughout two simulated solar cycles. This represents an ideal case situation that could arise if we had at all times 360 degrees of photospheric magnetogram data for the Sun. Next, we follow this with two "limited data" simulations. In the "Earth-only" simulation we include bipoles from the reference Sun simulation only when they are in the FOV from Earth. In the "Earth plus L5" simulation we increase this FOV in longitude to include that from a magnetograph at the L5 point. In this paper, the first of two, we focus on the simulation technique and on the time evolution of global properties of the magnetic field. These properties include the surface flux, volume-integrated magnetic energy, volume-integrated electric current, open flux, and number of nonerupting flux ropes present in all these simulations. In a second paper we will consider more localized properties such as the spatial and temporal distribution of free magnetic energy, along with the location and timing of flux rope ejections.

The paper is structured as follows. In Section 2 the global nonpotential evolution model is described, along with the distribution of bipoles used to simulate the varying level of magnetic activity over two solar cycles. Two cycles are considered as this gives the minimum time to consider the full cyclic evolution of the polar fields. In Section 3 a description of the reference Sun simulation is given, where bipole emergences can occur randomly at any longitude at any given time. The technique used to produce the limited data simulations from both the Earth-only and the Earth plus L5 viewpoints is described in Section 4. Results of the limited data simulations are then compared to the reference Sun simulation in Section 5. Finally, a discussion of the results, a description of future work, and the conclusions are given in Section 6.

\section{THE COMBINED MODEL}

A combination of magnetic flux transport (Sheeley 2005) and magnetofrictional relaxation simulations (van Ballegooijen et al. 2000; Mackay \& van Ballegooijen 2006) are used to simulate the continuous nonpotential evolution of the Sun's 3D global magnetic field (Mackay \& Yeates 2012). The magnetic flux transport model is used to simulate the evolution of $B_{r}$ at the solar surface under the combined effects of differential rotation (Snodgrass 1983), meridional flow (Duvall 1979), surface diffusion (Leighton 1964), and flux emergence. Coupled to the magnetic flux transport model, the magnetofrictional model (Yang et al. 1986) considers the quasi-static evolution of the coronal magnetic field, as it tries to relax to a nonlinear force-free state, in response to the combined effects of surface transport and flux emergence.

An important aspect of these simulations is the bipole data set that is used to represent flux emergence and drive the variation in magnetic activity over the simulated cycles. Two possible methods can be used to specify the data. First, it may be drawn from observational samples obtained from synoptic magnetograms (Yeates 2014). Second, it can be derived from a synthetic data set that satisfies observed empirical relationships (van Ballegooijen et al. 1998). For the present study we choose the latter, since current observational data sets will have an observational bias built into them due to the fact that we can only observe a portion of the surface of the Sun at any one time. This occurs in particular when using synoptic maps, as in a single map time runs in the direction of decreasing longitude. Since we use synthetic bipole data sets, we have full control over the number and locations of bipoles emerging each day, which can then be varied in the limited data simulations.

In the combined models the Sun's large-scale magnetic field, $\boldsymbol{B}=\left(B_{r}, B_{\theta}, B_{\phi}\right)=\nabla \times \boldsymbol{A}$ is evolved forward in time through the magnetic induction equation, where $(r, \theta, \phi)$ have their usual meaning. Full details of each of the model components are given below. 


\subsection{Surface Flux Transport Model}

To describe the evolution of magnetic fields in the photosphere, the induction equation at $r=R_{\odot}$ is prescribed as a boundary condition for the time derivatives of the horizontal components of the vector potential $A_{\theta}$ and $A_{\phi}$ (therefore on $B_{r}$ ),

$$
\begin{gathered}
\frac{\partial A_{\theta}}{\partial t}=+u_{\phi} B_{r}-\frac{D}{r \sin \theta} \frac{\partial B_{r}}{\partial \phi}+S_{\theta}(\theta, \phi, t) \\
\frac{\partial A_{\phi}}{\partial t}=-u_{\theta} B_{r}+\frac{D}{r} \frac{\partial B_{r}}{\partial \theta}+S_{\phi}(\theta, \phi, t),
\end{gathered}
$$

where $D$ is the photospheric diffusion constant $\left(D=450 \mathrm{~km}^{2}\right.$ $\mathrm{s}^{-1}$; see DeVore et al. 1985), $u_{\phi}$ is the azimuthal velocity, and $u_{\theta}$ is the meridional flow velocity. The azimuthal velocity is taken to be of the form

$$
u_{\phi}=\Omega(\theta) r \sin \theta,
$$

where $\Omega(\theta)$ is the angular velocity of differential rotation relative to the Carrington frame, which rotates at 13.20 deg day $^{-1}$ (Snodgrass 1983),

$$
\Omega(\theta)=0.18-2.30 \cos ^{2} \theta-1.62 \cos ^{4} \theta \text { deg day }{ }^{-1} .
$$

The poleward meridional flow is chosen to be of the form

$$
u_{\theta}=-C \sin (2 \lambda) \exp (\pi-2|\lambda|),
$$

where $C=15 \mathrm{~m} \cdot \mathrm{s}^{-1}$ and $\lambda=\frac{\pi}{2}-\theta$, which is taken from the paper by Schüssler \& Baumann (2006). The terms $S_{\theta}$ and $S_{\phi}$ are source terms that represent the emergence of new magnetic flux. Full details of how these source terms are specified are given in Section 2.3.

\subsection{Coronal Evolution Model}

The magnetic field within the coronal volume evolves in response to motions that occur in the photosphere through the induction equation,

$$
\frac{\partial \boldsymbol{A}}{\partial t}=\boldsymbol{v} \times \boldsymbol{B}+\frac{\boldsymbol{B}}{B^{2}} \nabla \cdot\left(\eta_{4} B^{2} \nabla \alpha\right),
$$

where $\boldsymbol{v}(\boldsymbol{r}, t)$ is the plasma velocity, $\alpha=\boldsymbol{B} \cdot \boldsymbol{j} / B^{2}$ is the current helicity, $\boldsymbol{j}=\nabla \times \boldsymbol{B}$, and $\eta_{4}=1 \times 10^{11} \mathrm{~km}^{4} \mathrm{~s}^{-1}$ is the coefficient of hyperdiffusion. We assume that the coronal plasma velocity is given by

$$
\boldsymbol{v}=\frac{1}{\nu} \frac{\boldsymbol{j} \times \boldsymbol{B}}{B^{2}}+v_{o} e^{-\left(2.5 R_{\odot}-r\right) / r_{w}} \hat{\boldsymbol{r}} .
$$

The first term on the right-hand side is the magnetofrictional velocity (Yang et al. 1986) and reflects the fact that in the corona the Lorentz force is dominant (low beta condition). The effect of this "frictional" term is that, when any field departs from a force-free state-as a result of boundary driving, for example - the magnetic forces in the corona act to return the field to a force-free state (generally, a nonlinear force-free field). The second term represents a radial outflow velocity that is imposed to ensure that the field lines remain radial at the source surface $\left(r=2.5 R_{\odot}\right)$. In a crude manner, this outflow velocity simulates the effect of the solar wind in opening coronal field lines. Its peak value is chosen to be $v_{o}=100 \mathrm{~km}$ $\mathrm{s}^{-1}$, and its exponential fall-off length from the outer boundary is $r_{w}=0.1 R_{\odot}$. This term is negligible in the low closed-field corona.

The second term in Equation (4) represents hyperdiffusion (van Ballegooijen \& Cranmer 2008), which is a higher-order form of diffusion that preserves magnetic helicity density within the coronal volume and tries to relax the magnetic field to a constant $\alpha$ (linear force-free) state (Boozer 1986; Bhattacharjee \& Hameiri 1986). For the simulations presented here, due to the constant stressing of the coronal field along with the long relaxation time of hyperdiffusion, such a constant $\alpha$ state is never reached.

To carry out the computations, positions within the domain are described in terms of new variables $x, y, z$ such that $x=\phi, y=-\ln [\tan (\theta / 2)], z=\ln \left(r / R_{\odot}\right)$ with a resolution of $\delta \phi$ (in heliographic degrees). To reduce computational overheads, a variable resolution is used, where at the equator the resolution is $\delta \phi=1.875$, which corresponds to 192 cells in $\phi$ and 28 in radius. The resolution is refined 4 times in each hemisphere, from the equator to the pole, such that the number of cells in $\phi$ reduces to 12 at the poles (see Yeates 2014). The simulated domain is $1 R_{\odot} \leqslant r \leqslant 2.5 R_{\odot}, 0.00873 \leqslant \theta \leqslant 3.133$, and $0 \leqslant \phi \leqslant 2 \pi$.

For the simulations presented here, the driving of the photospheric field and relaxation of the coronal field are carried out simultaneously. Due to this, the coronal field never strictly satisfies the force-free condition, but departs from it by only a small amount. Throughout the simulation the angle between $\boldsymbol{B}$ and $\boldsymbol{j}$ is monitored to ensure that it remains at a value that is less than a few degrees. With this procedure, a sequence of coronal quasi-equilibrium states are produced.

\subsection{Bipole Data Set}

To produce realistic simulations over two solar cycles of the Sun's surface and nonpotential coronal magnetic field, realistic input data are required to describe the emergence of new bipolar active regions. For the present study, only large-scale emergences of magnetic flux $\left(\geqslant 10^{20} \mathrm{Mx}\right)$ are considered. These bipoles follow an approximate $11 \mathrm{yr}$ cyclic variation, and as they interact with one another, they produce large unipolar areas that extend across the solar surface toward the poles.

To describe the emergence of new magnetic bipoles, the source terms $S_{\theta}$ and $S_{\phi}$ in Equations (1) and (2) have to be specified. Rather than choosing an explicit functional form for $S_{\theta}$ and $S_{\phi}$, we insert idealized bipoles whose properties are derived from observationally derived relationships (Wang \& Sheeley 1989; Tian et al. 1999). These properties include latitude of emergence, flux, area, tilt angle and number of bipoles emerging per day. For the latter quantity we specify the emergence rate as $a(t) A^{-2} d A$, where $A$ is the area of the bipole. The function $a(t)$ reproduces the varying levels of activity over the solar cycle (Harvey \& Zwaan 1993; Schrijver \& Harvey 1994). In addition to these properties, bipoles may emerge at any longitude at any given time.

Each bipole is inserted in an idealized form, both at the photosphere and in the corona. The 3D mathematical form of the bipole in terms of the coordinates $\left(x_{1}, y_{1}, z_{1}\right)$ relative to its 
center point is

$$
\begin{aligned}
& B_{x}=B_{0} e^{0.5}\left(\frac{z_{1}}{\rho_{0}} e^{-\xi}+4 \beta \frac{x_{1} y_{1}}{\rho_{0}^{2}} e^{-2 \xi}\right), \\
& B_{y}=2 \beta B_{0} e^{0.5}\left(1-\frac{x_{1}^{2}+z_{1}^{2}}{\rho_{0}^{2}}\right) e^{-2 \xi}, \\
& B_{z}=B_{0} e^{0.5}\left(-\frac{x_{1}}{\rho_{0}} e^{-\xi}+4 \beta \frac{y_{1} z_{1}}{\rho_{0}^{2}} e^{-2 \xi}\right),
\end{aligned}
$$

where $\rho_{0}$ is the half separation between the peaks of the photospheric flux patterns, $B_{0}=\Phi /\left(\sqrt{\pi e} \rho^{2}\right)$ is the peak flux density, $\Phi$ is the total flux, $\xi \equiv\left[\left(x_{1}^{2}+z_{1}^{2}\right) / 2+y_{1}^{2}\right] / \rho_{0}^{2}$, and $\beta$ is a dimensionless parameter describing the self-twist of the bipole. For the present simulations, which consider only the possibility of a normal component magnetograph at the L5 point, we assume that $\beta=0$. This is because such an instrument would not be able to provide information on the horizontal components of the field inside active regions. It should, however, be noted that while the self-helicity of each bipole is zero, when the bipole is added to the coronal field there is a mutual helicity between it and the 3D coronal field. The sign of the mutual helicity is not prescribed and depends on the relative orientation of the bipole to the overlying field. The insertion of each magnetic bipole into the 3D coronal field is done in terms of the vector potential $\boldsymbol{A}$, so that the condition $\nabla \cdot \boldsymbol{B}=0$ is satisfied exactly. The bipoles may also be rotated by a given tilt $(\gamma)$ consistent with Joy's law.

Figure 1 shows the main properties of the magnetic bipoles that emerged during the two simulated solar cycles. These bipoles are used as the bipole data set in the reference simulation described in Section 3. Figure 1(a) shows a plot of the bipoles' latitude of emergence as a function of time (in yr). Over the two simulated cycles, the emergence latitudes of the bipoles produce a pattern very similar to the solar butterfly diagram of sunspots. Over the two cycles a total of 4725 magnetic bipoles emerge, containing a total flux of $3.21 \times 10^{25} \mathrm{Mx}$. The number of bipoles emerging every 27 days can be seen in Figure 2 (blue line). At cycle maximum approximately 1.2 bipoles emerge per day, which decreases to around 4 per rotation at cycle minimum. These rates of emergence produce a modest level of magnetic activity, corresponding to that found in cycles 20 and 23 . In Figure 1(b) the scatter plot shows the relationship between the net magnetic flux of the bipoles and the half-width separation between the peaks in each polarity. The range of fluxes extends over 2 orders of magnitude where the relationship between flux and half separation closely resembles that found in Yeates (2014), who plotted the relationship for bipoles deduced from Kitt Peak synoptic magnetograms. Finally, in Figure 1(c) the relationship between the tilt angle $(\gamma)$ of the bipoles and their emergence latitude can be seen. Bipoles in the northern/southern hemisphere that emerge with positive/ negative tilt angles follow Joy's law. While there is a wide scatter in the plot, the relationship varies as $\gamma \sim 0.29 \lambda$. In the next section we will consider the properties of the reference Sun simulation when its photospheric and coronal fields are driven by the bipole data described above.

\section{REFERENCE SUN SIMULATION}

The reference Sun simulation is carried out using the combined surface flux transport and magnetofrictional models, along with all of the bipoles presented in Section 2.3. The results of this simulation, where the Sun's global nonpotential field is simulated for $22 \mathrm{yr}$, can be seen in Figures 3 and 4. Figure 3 shows the variation in a number of globally integrated quantities and includes (a) surface flux $\left(\Phi_{s}\right.$, Equation (5)), (b) total magnetic energy $\left(E_{m}\right.$, Equation (6)), (c) total magnitude of electric current ( $J_{V}$, Equation (7)), and finally (d) the open flux $\left(\Phi_{o p}\right.$, Equation (8)),

$$
\begin{gathered}
\Phi_{s}(t)=R_{\odot}^{2} \int_{s}\left|B_{r}\left(R_{\odot}, \theta, \phi, t\right)\right| d \Omega, \\
E_{m}(t)=\int_{V} \frac{B^{2}(r, \theta, \phi, t)}{8 \pi} d \tau, \\
J_{V}(t)=\int_{V}|j(r, \theta, \phi, t)| d \tau, \\
\Phi_{o p}=\left(2.5 R_{\odot}\right)^{2} \int_{s}\left|B_{r}\left(2.5 R_{\odot}, \theta, \phi, t\right)\right| d \Omega .
\end{gathered}
$$

Most of these quantities are commonly used; the volumeintegrated electric current gives a measure of the nonpotential state of the coronal field. From Figure 3, it can be seen that the surface flux, magnetic energy, and electric current all show a clear cyclic variation that varies in phase with the activity cycle. The surface flux exhibits the smallest variation (factor of 4) between cycle minimum and maximum, while the magnetic energy shows the strongest variation at nearly an order of magnitude. In contrast, the open flux exhibits the weakest variation (factor of 2) and does not show a clear cyclic behavior. Such a variation is, however, consistent with that found by Lockwood et al. (2004) for cycles 20 and 23. It is interesting to note that cycles 20 and 23 had a medium level of activity, similar to the cycles considered here. Spikes in the open flux occur around years 5 and 16, corresponding to cycle maximum in each cycle, which is again consistent with observations.

Figure 4(a) shows the variation of the north polar flux (solid line) and south polar flux (dashed line), where the (signed) polar flux is calculated for latitudes above $70^{\circ}$ in each hemisphere. At the start of each cycle (years 0 and 11) the polar fields are of opposite sign, but reverse midway through each cycle, consistent with observations and when the spikes in open flux occur. In addition, the quantity of flux in the polar regions remains consistent at the start and end of each cycle. The bipole properties chosen in Section 2.3 therefore produce a stable variation of the global magnetic field based on the amount of flux that exists in the polar regions and the strength of the cycle. Finally, in Figure 4(b) we see a graph of the number of flux ropes formed in the simulation throughout the two solar cycles. While the previous graphs in Figures 3 and 4(a) are sampled once per day, due to the time-consuming nature in identifying flux ropes, Figure 4(b) is sampled once every 50 days. It is clear that the number of flux ropes varies in phase with the simulated cycles. At cycle minimum the number of flux ropes varies from 4 to 7 , while at cycle maximum it can be as high as 20-25. This variation is important for space weather, as these flux ropes can become unstable and be ejected from the simulation, resulting in a CME (Pagano et al. 2013). It should be noted that currently we are only counting the number 


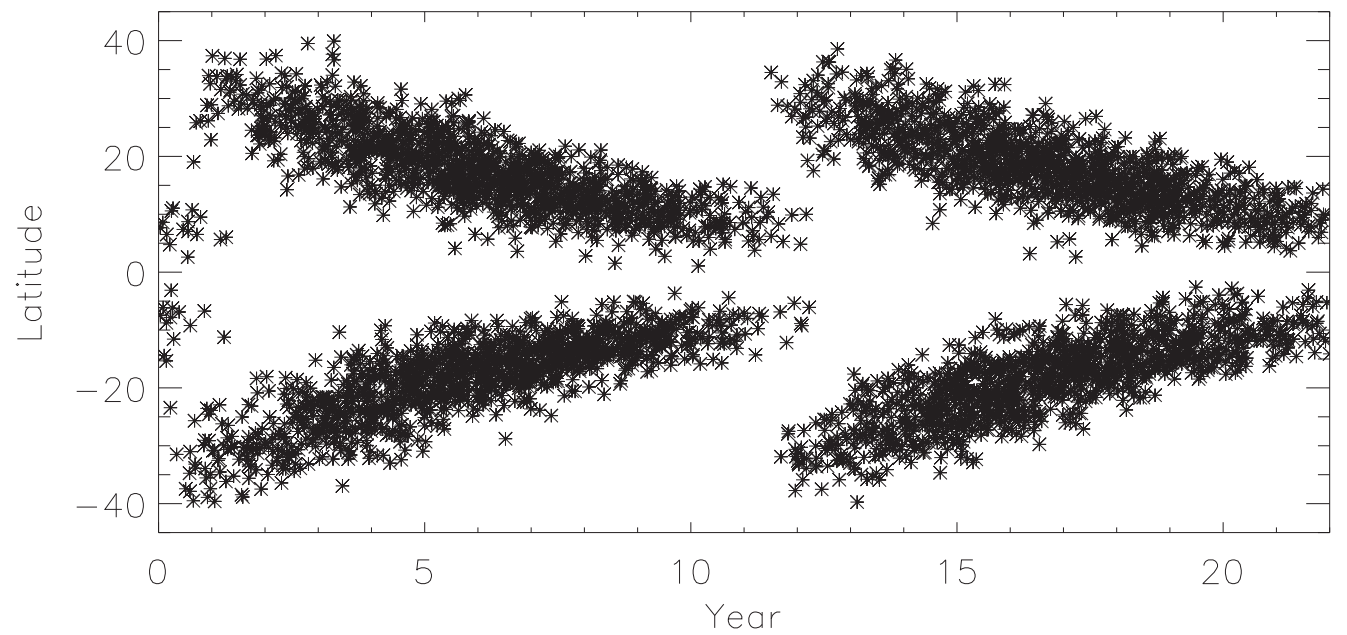

(a)

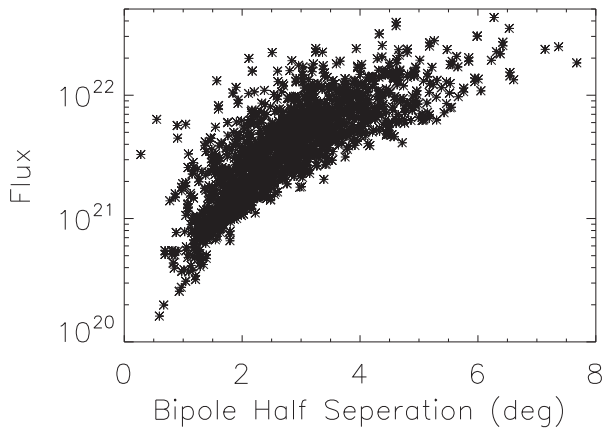

(b)

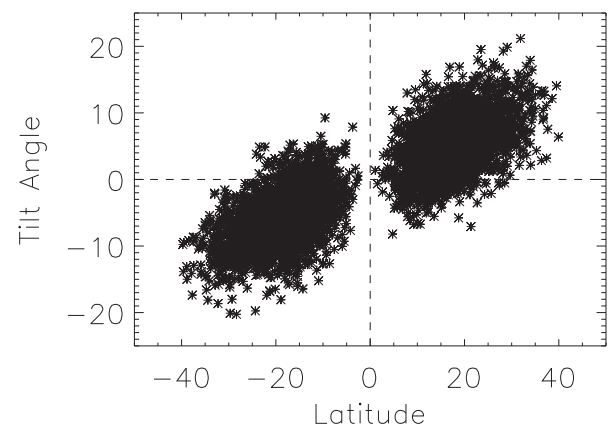

(c)

Figure 1. Properties of the input data used in the reference Sun simulation over 22 yr. (a) Butterfly diagram. (b) Graph of bipole flux (Mx) against half separation (degrees) of the peaks in the bipole polarities. (c) Graph of tilt angle (degrees) against latitude. The two dashed lines denote zero tilt angle and zero latitude.

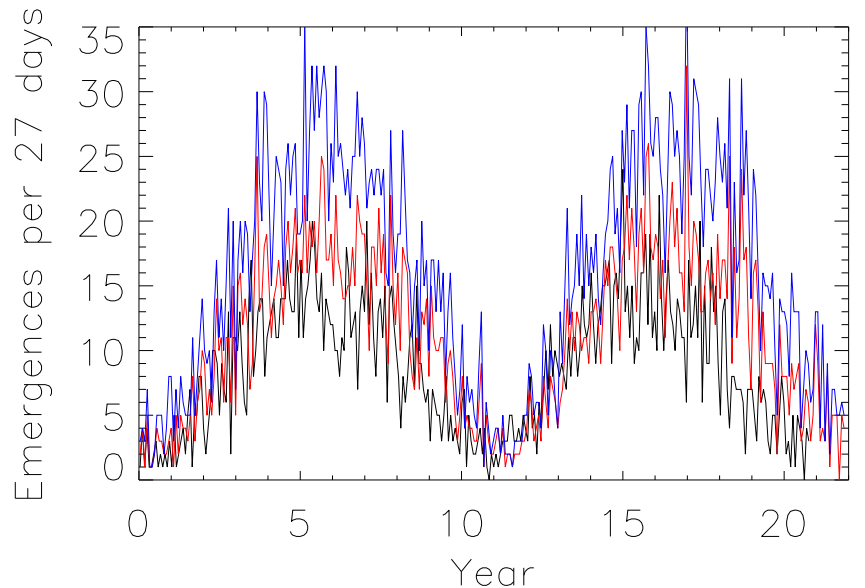

Figure 2. Graphs of the number of bipoles emerging over every 27-day period vs. time in yr. The blue line represents the reference Sun simulation, black the Earth-only simulation, and red the Earth plus L5 simulation.

of stable flux ropes and are not including those in the process of erupting. To consider the latter, an identification and crosscorrelation analysis of the simulated 3D coronal magnetic field at a time cadence of less than a day are required to count and track the flux ropes as they progress through the solar corona. Such an in-depth study is beyond the scope of the present paper. However, a future study that will undertake this is described in Section 6.

Within the global nonpotential simulation we have significant freedom in producing either a stronger or weaker variation in each of the above quantities. This can be achieved by increasing or decreasing the rate of bipole emergence, or by introducing a nonzero bipole twist parameter. The chosen parameters do, however, show a clear solar cycle variation, consistent with observations. For the remainder of the paper we will only consider how well the limited data simulations from either Earth or Earth plus L5 reproduce these results. Thus, in later graphs we will not show absolute values, but rather (usually) ratios of values.

\section{LIMITED DATA SIMULATIONS}

\subsection{Description of Technique}

In Section 3, the reference Sun simulation provides the optimal case of what the global nonpotential model can produce if we have magnetic field observations providing full coverage of the solar surface. While full coverage of the surface field is desirable, at the present time we have a limited FOV. We now need to consider what effect this limited FOV has on the accuracy of the limited data simulations and how having observations at the L5 point may improve their accuracy. An illustration of the effect of this FOV can be seen in Figure 5. In each of the plots the dashed line represents the central meridian 


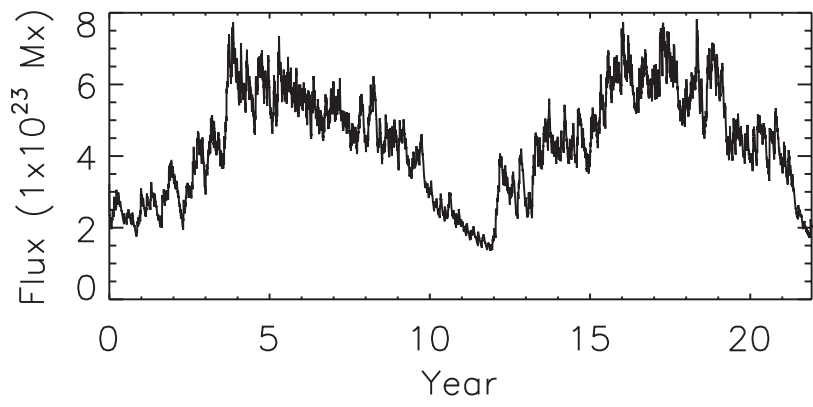

(a)

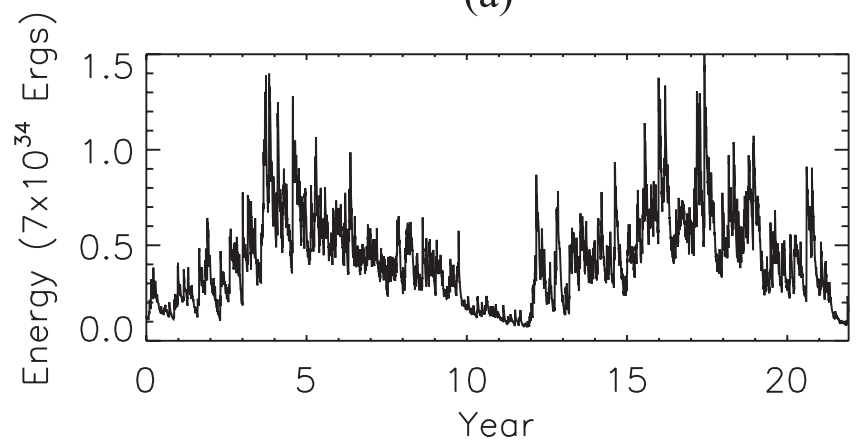

(b)

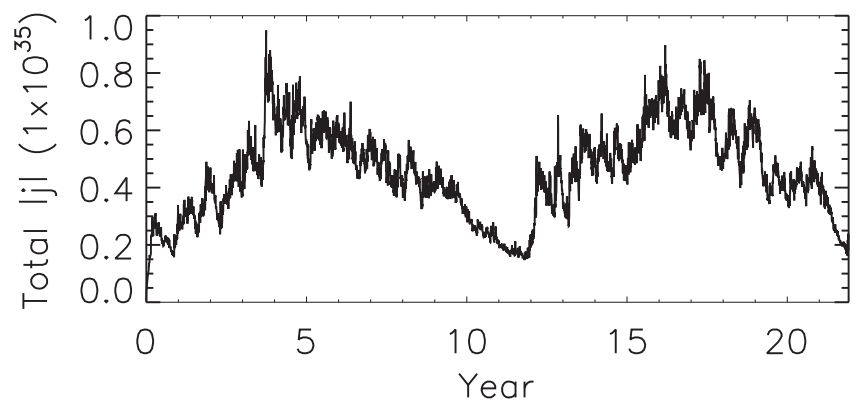

(c)

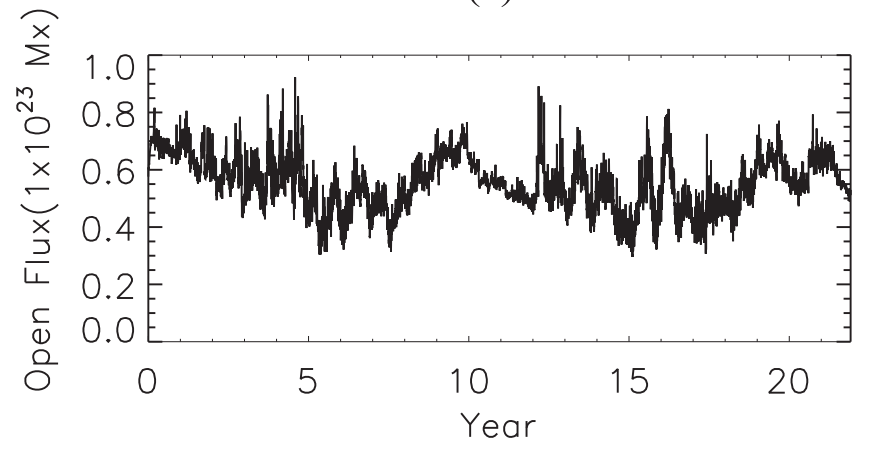

(d)

Figure 3. Graphs showing the properties of the reference Sun simulation as a function of time in yr. They show the variation of (a) surface flux, (b) volumeintegrated magnetic energy, (c) volume-integrated $|\boldsymbol{j}|$, and (d) open flux.

longitude of the Sun as seen from Earth $\left(\mathrm{CM}_{\mathrm{E}}\right)$ at $5.12 \mathrm{yr}$ into the simulation. The two dot-dashed lines denote the boundary lines for the FOV of observations. They give locations where, due to LOS effects, magnetic field measurements are regarded as no longer accurate. These are shown both ahead and behind $\mathrm{CM}_{\mathrm{E}}$. For the present simulations we choose the boundary where measurements are no longer regarded as accurate to extend

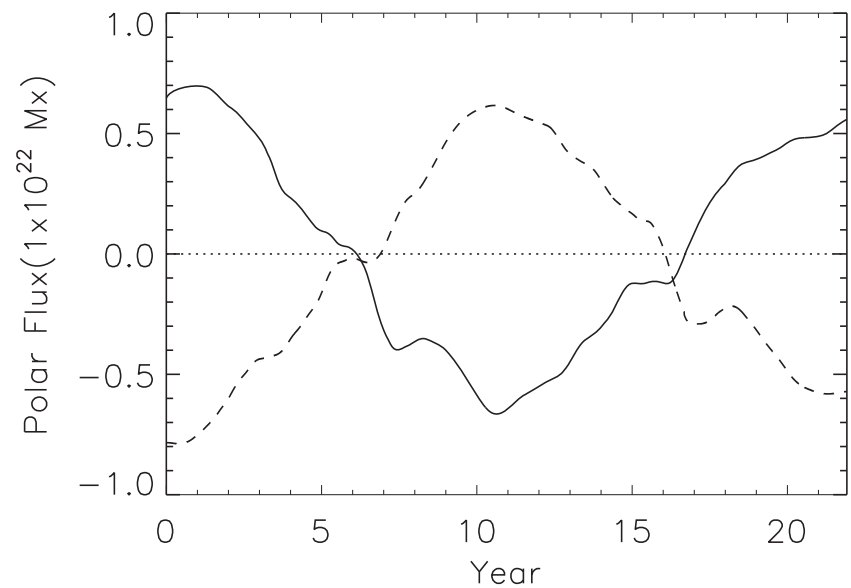

(a)

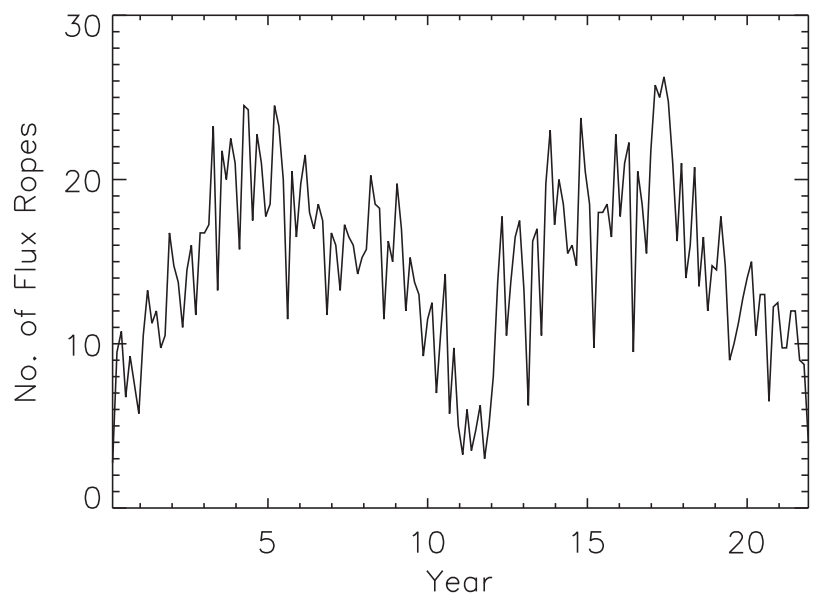

(b)

Figure 4. (a) Variation of the polar field strengths in the reference Sun simulation for both the northern (solid line) and southern (dashed line) hemispheres. The dotted line denotes the zero value. (b) Variation in the number of nonerupting flux ropes in the reference Sun simulation.

up to $30^{\circ}$ from the limb. Beyond this limit magnetograph data can suffer from a number of issues such as foreshortening, instrument noise, and the canopy effect. While the choice of $30^{\circ}$ from the limb can be varied slightly, there are strong arguments against using data that lie within $15^{\circ}$ of the limb (Worden \& Harvey 2000). So although the placement of the boundary relative to the limb can be varied $\left(\leqslant 10^{\circ}\right)$, any such variation would only increase/decrease the accuracy of both of the limited data simulations by the same amount. The relative difference between the two limited data simulations would be unchanged. For the present simulations, it is assumed that any bipolar magnetic region that lies within the FOV enclosed by the two dot-dashed lines will be observed accurately enough that it may be included in the global nonpotential model.

The Earth-only (Figure 5(a)) and Earth plus L5 (Figure 5(b)) FOVs (yellow areas) share the same leading boundary, since we do not allow for a magnetograph at the $\mathrm{L} 4$ point $\left(60^{\circ}\right.$ ahead of the Earth in its orbit). However, the L5 magnetograph increases the FOV by $60^{\circ}$ at the trailing boundary, so that the FOV is $\left[\mathrm{CM}_{\mathrm{E}}-120^{\circ}, \mathrm{CM}_{\mathrm{E}}+60^{\circ}\right]$ for the Earth plus L5 simulation, as opposed to $\left[\mathrm{CM}_{\mathrm{E}}-60^{\circ}, \mathrm{CM}_{\mathrm{E}}+60^{\circ}\right]$ for the Earth-only simulation. Note 


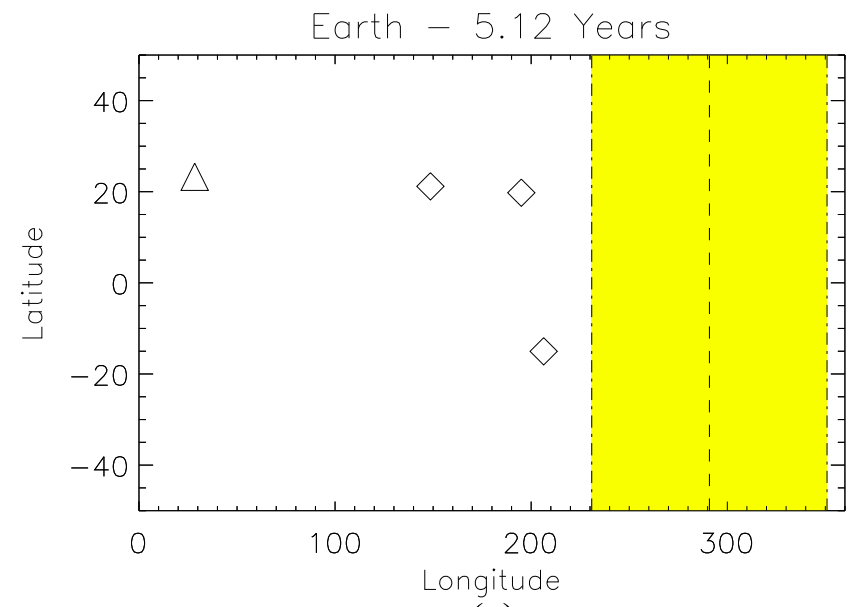

(a)

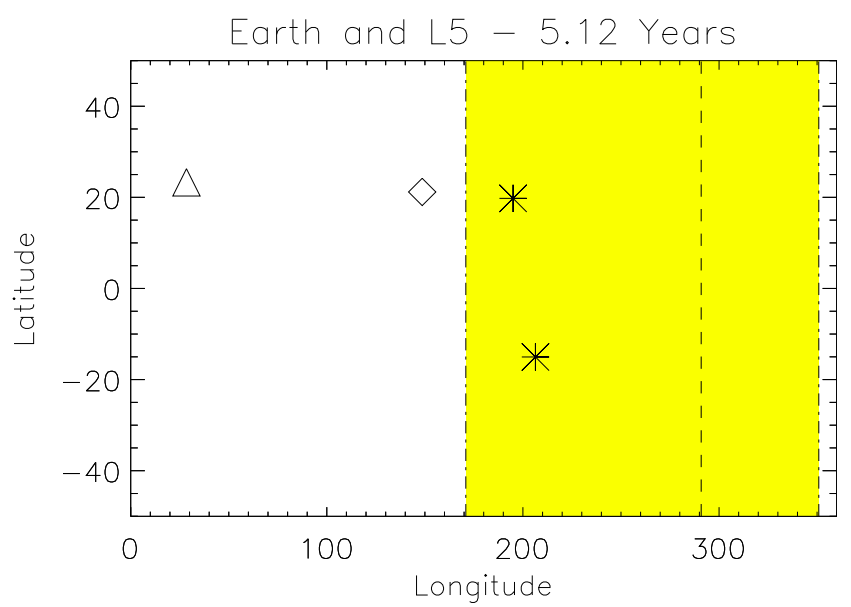

(b)

Figure 5. Plots of the location of the emergence of new magnetic bipoles relative to the FOV (yellow) for (a) Earth-only observations and (b) Earth plus L5 observations at $5.12 \mathrm{yr}$ into the simulation. In each plot the dashed line denotes the location of central meridian of the Sun (at $290^{\circ}$ longitude) as seen from Earth, while the dot-dashed lines denote the boundaries for the FOV. Bipoles given by stars emerged in real time; diamonds are included late, once they rotate into the FOV; and triangles are missed completely.

that these boundaries are not fixed, but change due to the rotation of the Sun.

The symbols in each plot of Figure 5 show the locations where four bipoles emerge within the reference Sun simulation on this day, three in the northern hemisphere and one in the southern hemisphere. From Figure 5(a) it is clear that in the Earth-only simulation none of these bipoles emerge within its FOV. Due to this, these bipoles are not included in the simulation in "real time," i.e., at the actual time when they emerge. This simulates the present situation with only Earthbased magnetograph observations. In contrast, for the Earth plus L5 simulation two of the bipoles $\left(\phi=195^{\circ}, \lambda=20^{\circ}\right.$ and $\phi=206^{\circ}, \lambda=-15^{\circ}$ ) lie within the extended FOV that occurs as a consequence of the L5 magnetograph. Both are indicated by the stars and emerge at the same time, with the same properties, as in the reference Sun simulation. This improves the accuracy of the Earth plus L5 simulation relative to the Earth-only simulation. For the Earth plus L5 simulation the other two bipoles are not included in real time as they lie outside its FOV.
For any future space weather prediction model, the best way to accurately represent the global magnetic field of the Sun will be to include as many magnetic bipoles in real time as possible. This would then allow us to follow the full dynamics of the field and the injection of Poynting flux into the corona. As Figure 5 illustrates, at the present time it is impossible to include all regions in this way, as we do not have $360^{\circ}$ coverage of the Sun with magnetograph observations. In future, helioseismology and far-side imaging techniques may play an important role in producing accurate proxy measurements for including far-side bipoles. It is, however, important that all models simulate the Sun as accurately as possible. Therefore, when bipoles emerge outside the observational FOV, but subsequently rotate into it, they should be included in the model with the earliest possible determination of their properties.

To represent this, we have also run simulations including what we call "rotational updates." These are additional bipoles that emerged outside the FOV but subsequently rotated into it. Since we know the properties of every bipole at the time of emergence (from the reference Sun simulation), it is convenient to evolve these properties forward in time to generate appropriate properties for the time when bipoles enter the FOV. In a real data-driven model, the properties of such bipoles would have to be determined from observations only once they rotated into the FOV. It should be noted that this rotational update procedure will not produce the same coronal field as we would get from inserting the bipoles at their original emergence times. Bipoles added later will have lower energy, owing to the shorter time for building up stress in the evolving corona. Nevertheless, we might hope that including such rotational updates will improve the simulations and better represent the real data-driven situation.

To evolve the bipole properties forward in time, we apply a simple model. This model updates the bipole properties due to their forward evolution in time, under the flux transport processes, to the point where the bipole crosses the boundary of the FOV and can be included in the simulation. The bipole properties that are updated include central bipole longitude $\left(\phi_{c}\right)$, central bipole co-latitude $\left(\theta_{c}\right)$, tilt angle $(\gamma)$, separation $(\rho)$, and flux $(\Phi)$. The first four values may be updated from knowing their original values, the differential rotation profile, and finally the time of evolution. They follow the following equations:

$$
\begin{gathered}
\theta_{c}(t)=\theta_{c}(0):=\theta_{c}, \\
\phi_{c}(t)=\phi_{c}(0)+\frac{\Omega\left(\theta_{1}\right)+\Omega\left(\theta_{2}\right)}{2} t, \\
\tan \gamma(t)=\frac{2 \rho(0) \sin \gamma(0)}{2 \rho(0) \cos \gamma(0)+r d \Omega t \sin \theta_{c}}, \\
\rho(t)=\sqrt{d_{1}+d_{2}} .
\end{gathered}
$$

where

$$
\begin{aligned}
d_{1} & =\left(\frac{\rho(0) \cos \gamma(0)}{r \sin \theta_{c}}+\frac{d \Omega}{2} t\right)^{2} r^{2} \sin ^{2} \theta_{c}, \\
d_{2} & =\rho^{2}(0) \sin ^{2} \gamma(0), \\
d \Omega & =\Omega\left(\theta_{1}\right)-\Omega\left(\theta_{2}\right),
\end{aligned}
$$

and $\theta_{1}$ and $\theta_{2}$ are the co-latitudes of the leading and following polarities. It is assumed that over short periods of time the effect of meridional flow on these quantities is negligible. Hence, $\theta_{c}, \theta_{1}$, and $\theta_{2}$ remain fixed for all time. While it is 
Table 1

Number of Magnetic Bipoles Included in the Limited Data Simulations as Real-time Emergences or Rotational Updates, and Number Missing

\begin{tabular}{lccc}
\hline \hline Simulation & Real Time & $\begin{array}{c}\text { Rotational } \\
\text { Updates }\end{array}$ & Missing \\
\hline Earth only & $1427(30.2 \%)$ & $1152(24.4 \%)$ & $2146(45.4 \%)$ \\
Earth plus L5 & $2140(45.4 \%)$ & $1178(24.9 \%)$ & $1407(29.7 \%)$ \\
\hline
\end{tabular}

Note. The number in parentheses gives the percentage of the reference Sun population.

straightforward to compute these quantities, the variation of the flux in the bipole is more difficult. One possible way would be to simulate each bipole individually. While such a method is possible, due to the number of bipoles that we would need to consider (2000-3000), we instead construct an analytical model to determine the change in flux (Yeates 2009). This model is described fully in the Appendix, where it is determined using a local planar geometry in a Lagrangian frame of reference. The variation of flux with time is then

$$
\Phi(t)=B_{o} \rho(0)^{3} \sqrt{2 \pi e} \sqrt{\frac{W(t)}{Q(t)}},
$$

where the functions $W(t)$ and $Q(t)$ depend on the initial bipole properties-full expressions are given in the Appendix. This model for advancing the bipole properties in time assumes that the bipole evolves on the Sun as an isolated region, with no interaction or cancellation with surrounding fields. On careful study of the reference Sun simulation, for a number of bipoles that emerge during varying levels of activity, it is found that advancing the properties forward in time as an isolated region produces only minor differences in the majority of cases. While we alter the above properties of the bipoles, since we are assuming that only an LOS magnetograph is present at the L5 point, we simply fix $\beta=0$.

In order to include the rotational updates, we only consider bipoles that emerge and rotate across the boundary behind central meridian within 7 days of emergence. This is an arbitrary choice, but is used to represent the fact that some regions may emerge and fragment before they return to the visible side of the Sun and are thus no longer in a coherent form and cannot be included in the model as a bipole. Varying this 7day time frame has a similar effect to varying the FOV boundaries, where the overall agreement between the limited data simulations and reference Sun simulation will either increase or decrease. However, we once again note that the relative difference between the two limited data simulations will remain the same. Bipoles that are included as rotational updates are denoted by the diamonds in Figure 5. For the Earthonly simulation, three bipoles emerged with updated properties $1.22,2.0$, and 5.6 days after they emerge in the reference Sun simulation. For the Earth plus L5 simulation only one region emerged 1.5 days late. For the bipole emergence pattern seen in Figure 5, a consequence of the rotational updates is to reduce the difference between the Earth-only and Earth plus L5 simulations, as in both cases three bipoles emerged, albeit at different times and with different properties. The Earth plus L5 simulation should, however, be the most accurate, as more of its bipoles will occur in real time. For both of the limited data simulations the bipole located at $\phi=28^{\circ}, \lambda=23^{\circ}$ is missed

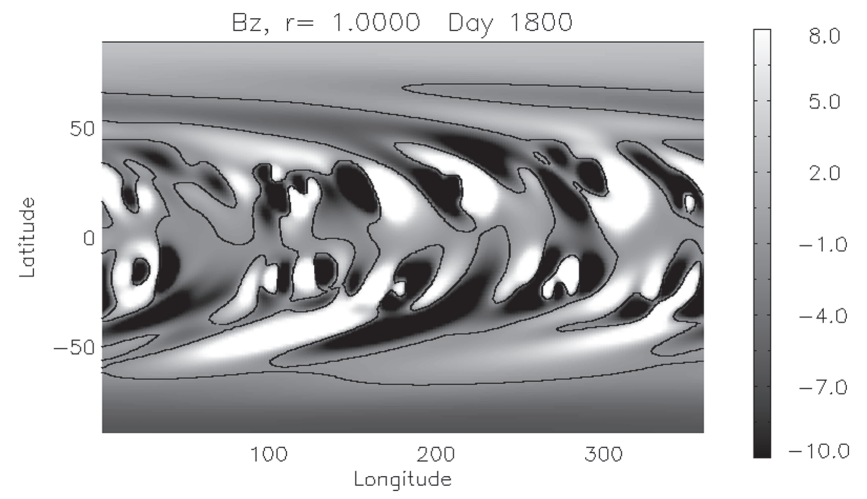

Figure 6. Illustration of the radial magnetic field distribution on day 1800 for the reference Sun simulation. White/black represents positive/negative flux, where the bar on the right-hand side gives the scale. The thin black line denotes the PIL.

as it lies beyond the 7-day period for inclusion as a rotational update.

In Table 1 we can see the number of bipoles that emerged in real time, as rotational updates, and missed for each of the limited data simulations. The number in parentheses gives the percentage that falls into each category, in terms of the total number of bipoles that emerged in the reference Sun simulation $(\sim 425)$. As expected, more bipoles are included as real-time emergences and rotational updates in the Earth plus L5 simulation (70.3\%) compared to the Earth-only simulation $(54.6 \%)$. It is also clear that rotational updates play an important role in each of the simulations. When they are not included, the activity level in the limited data simulations is less than half that in the reference Sun simulation.

The number of bipoles emerging in both real time and as rotational updates over every 27-day period can be seen in Figure 2. Blue represents the reference Sun simulation, black the Earth-only simulation, and red the Earth plus L5 simulation. From this it can be seen that each of the simulations follows a clear cyclic evolution over the two cycles.

\section{COMPARISON OF LIMITED DATA AND REFERENCE SUN SIMULATIONS}

In this section we now consider a detailed comparison of the reference Sun and limited data simulations, to consider how well the latter reproduce the global quantities found in the reference Sun simulation. In the present study it is assumed that both limited data simulations have the same initial condition as that of the reference Sun simulation, both for the photospheric field and for the coronal field, which is assumed to be potential. We will also determine what improvements occur when L5 observations are included, compared to when they are missing. To begin with, we consider first the accuracy of the spatial distribution of the radial magnetic field at the photosphere (Section 5.1) and then a number of global integrated quantities (Section 5.2).

\subsection{Photospheric Field}

In Figure 6 an illustration of the photospheric radial magnetic field for the reference Sun simulation can be seen at $4.93 \mathrm{yr}$ into the simulation. The date is chosen to lie within the first simulated cycle during the rising phase, close to cycle maximum. It can be seen that there is a large amount of 


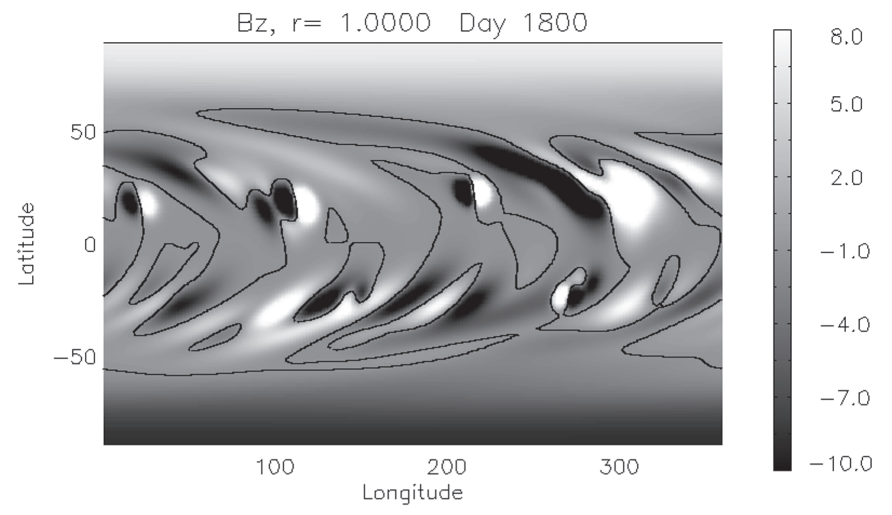

(a)

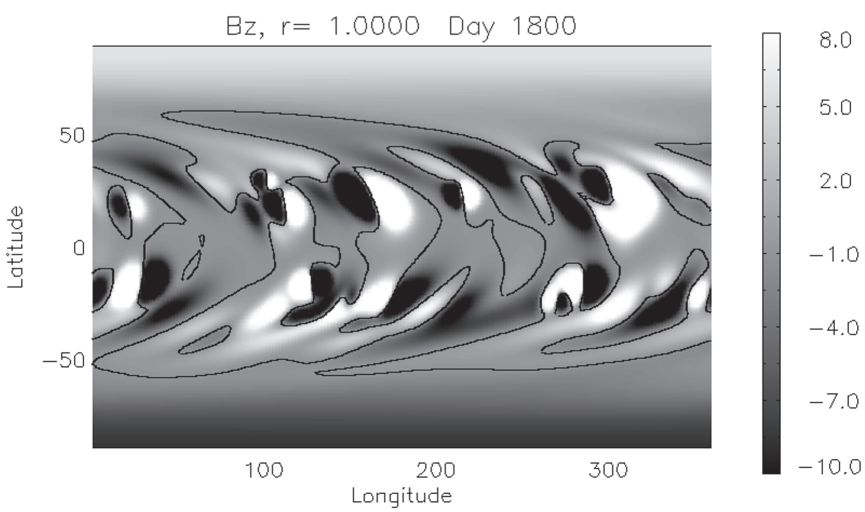

(b)

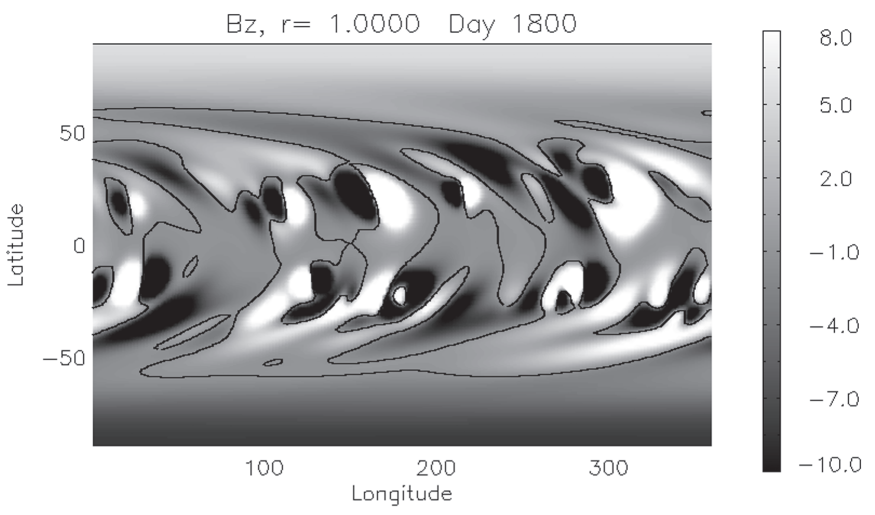

(c)

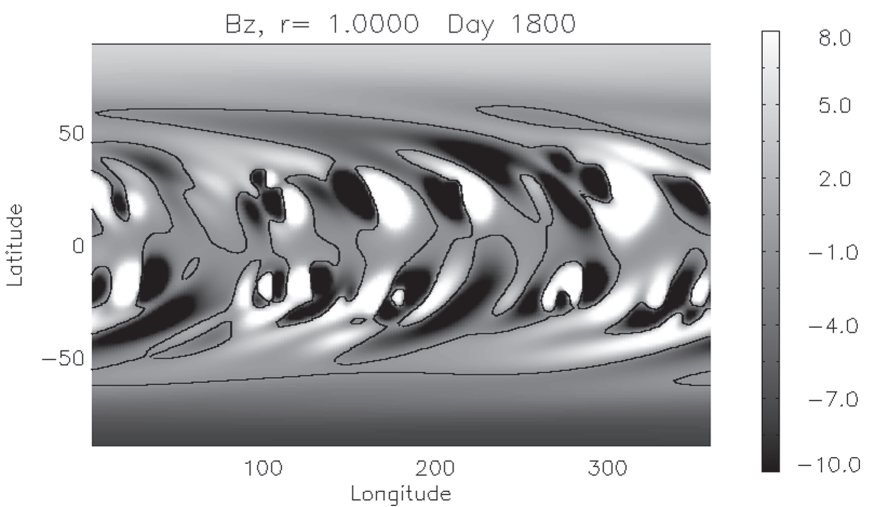

(d)

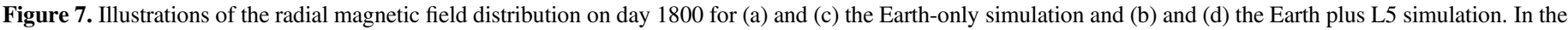

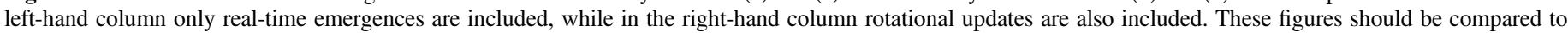
Figure 6. For each of the plots white/black represents positive/negative flux, where the bar on the right-hand side gives the scale.

magnetic flux at low latitudes due to the high level of activity. In each hemisphere at latitudes greater than $60^{\circ}$ there is a distinct polar crown polarity inversion line (PIL). The polar crown PIL is fully formed in the southern hemisphere, where it completely encloses the polar cap. However, in the northern hemisphere it is broken. The formation of these east-west polar crown PILs illustrates the slow transit to the poles in each hemisphere of the trailing polarity flux from the active latitudes, under the influence of meridional flow. This flux first cancels with the polar field and then reverses the polarity of the polar field just after cycle maximum (see Figure 4).

In Figure 7 the images show the photospheric field at the same time for (a) and (c) the Earth-only simulation and (b) and (d) the Earth plus L5 simulation. For illustrative purposes the left-hand column shows cases where only real-time emergences are included. The right-hand column gives full simulation results when both real-time emergences and rotational updates are included. These images show typical results found throughout the simulation. For the simulations where only real-time emergences are included, the Earth-only simulation (Figure 7(a)) shows limited agreement with the reference Sun simulation (Figure 6). At low latitudes a significant number of the bipoles are missing, with much lower levels of activity seen. It is clear that due to the limited FOV and number of bipoles that emerged, the simulation has not captured the complexity of the reference Sun simulation. In Figure 7(b), where the L5 observations are included, we see a much higher level of activity at low latitudes where it better reproduces the reference Sun simulation (Figure 6). Visually at least, the Earth plus L5 simulation produces a much better result. This illustrates the importance of including observations from the L5 point.

The effect of including the rotational updates along with the real-time emergences can be seen in Figure 7 for (c) the Earthonly simulation and (d) Earth plus L5. It is clear from these images that with the inclusion of real-time emergences and rotational updates, both of the limited data simulations produce much more realistic results. At low latitudes and any given longitude, it is now difficult to distinguish the two limited data simulations from each other and from the reference Sun simulation. However, as expected, the Earth plus L5 case produces better results, where more distinct differences can be seen in the higher-latitude field. The Earth plus L5 case reproduces more accurately the polar crown PILs in both hemispheres. In particular, in the southern hemisphere it has nearly produced a complete polar crown encircling the polar cap.

A more quantitative comparison of the simulations shown in Figures 7(c) and (d) is carried out in Figure 8, where the correlation between the limited data and reference Sun simulations is plotted for the cases that include both real-time emergences and rotational updates. The correlation tests the accuracy of the limited data simulations in producing the correct sign of the field at each pixel over the entire simulation. Due to the decreasing area toward the poles, the comparison is dominated toward the lower latitudes. In Figure 8, the solid line 


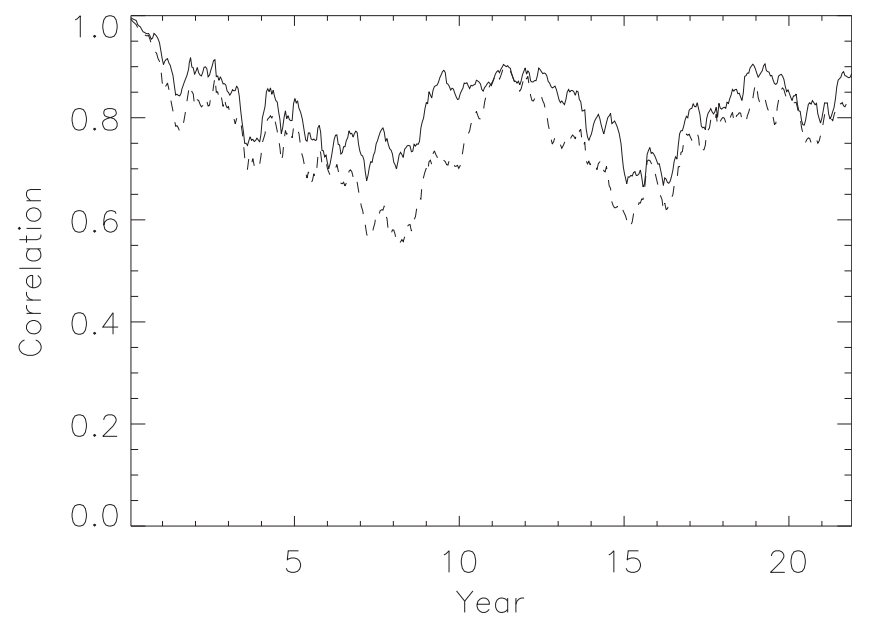

Figure 8. Correlation between the sign of the radial magnetic field at the photosphere in the reference Sun simulation and the Earth-only simulation (dashed line) or Earth plus L5 simulation (solid line). Results are shown for real-time plus rotational updates.

compares the Earth plus L5 simulation to the reference Sun simulation, while the dashed line compares the Earth-only simulation to the reference Sun simulation. After the initial phase lasting approximately $1 \mathrm{yr}$, where the correlation is high (as both simulations start with the same initial field), the correlation ranges from $70 \%$ to $90 \%$ for the Earth plus L5 case (solid line) and from $55 \%$ to $90 \%$ for the Earth-only case (dashed line). Both simulations produce the best correlation near cycle minimum when there is least magnetic flux emerging at low latitudes. The images shown in Figure 7 are given for $4.9 \mathrm{yr}$ into the simulation, where the correlation coefficient is 0.8 for the Earth plus L5 case and 0.7 for the Earth-only case. ${ }^{6}$ The Earth plus L5 simulation produces a better correlation especially during the declining phase of the first and rising phase of the second cycle.

In Figure 9(a) we can see the variation in polar flux throughout each $22 \mathrm{yr}$ simulation. In each case the solid line gives the variation of the north polar region above $70^{\circ}$ latitude and the dashed line the south polar region below $-70^{\circ}$ latitude. The blue curves give the results for the reference Sun simulation (as given in Figure 4(a)) and are included for reference. The black and red lines show the results for the Earth-only and Earth plus L5 cases, respectively. All simulations start with the same amount of polar flux as they have the same initial condition. In the reference Sun simulation it is clear that the polar fields reverse polarity just after cycle maximum in each cycle, with a small difference in reversal time between the northern and southern hemispheres. In both of the limited data simulations reversals of the polar field can be seen. The Earth plus L5 simulation (red line) produces closer results to the reference Sun simulation than the Earth-only case. While reversals are found, the polar fields produced by both limited data simulations are too weak to reproduce accurately the results of the reference Sun simulation. This leads in both cases to the first reversal occurring far too late in the cycle and produces too weak a polar field in the next cycle. This weak field can then be easily reversed. Thus, even though we include rotational updates along with the real-time emergences, insufficient flux is pushed poleward in order to cancel the

\footnotetext{
6 If only real-time emergences are included, the coefficients are 0.75 and 0.65 respectively.
}

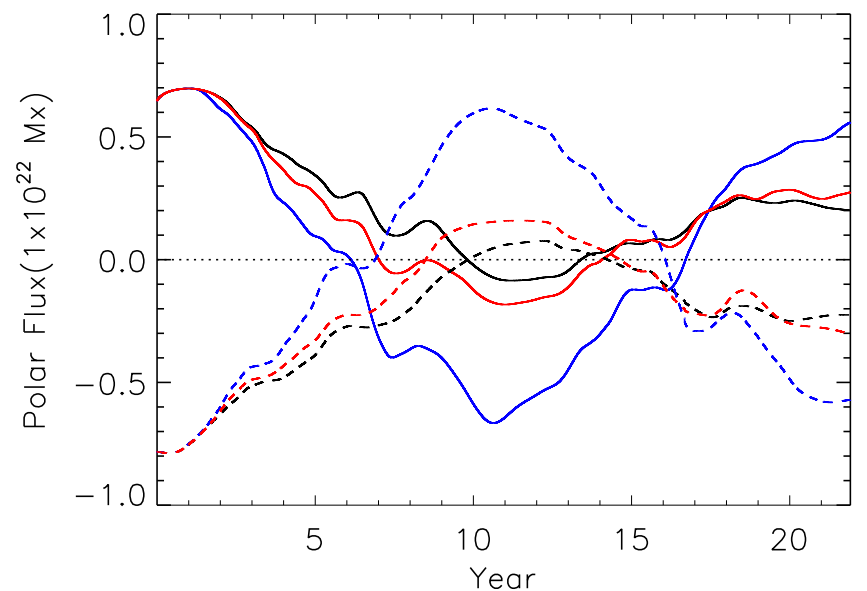

Figure 9. Variation of the polar fields for both the northern (solid line) and southern (dashed line) hemispheres. In each case the black lines denote the Earth-only simulation, the red lines the Earth plus L5 simulation, and the blue lines the reference Sun simulation. Each limited data simulation includes realtime emergences and rotational updates.

preexisting polar fields and build up fields of the correct strength at the correct time. This illustrates the sensitivity of the polar fields to the bipoles that are missed.

While the polar regions may only be a small portion of the solar surface, they do play a key role in determining the location and variation of the Sun's open flux, which is itself important for operational space weather models such as Enlil (Odstrcil \& Pizzo 1999). We will discuss this further when we consider the variation of the open flux in Section 5.2.

\subsection{Global Integrated Quantities}

We now consider how accurately the limited data simulations reproduce the global integrated quantities in the reference Sun simulation. In Figure 10 we can see a comparison for the simulations where both real-time emergences and rotational updates are considered. Each graph shows the ratio between the Earth-only simulation (black line) or Earth plus L5 simulation (red line) and the corresponding quantity from the reference Sun simulation. Since we are plotting the ratio, agreement between the limited data and reference Sun simulations is given by a value of 1 . The graphs are plotted for ratios of (a) surface flux $\Phi_{s}$, (b) volume-integrated magnetic energy $E_{m}$, and (c) volume-integrated electric current density $J_{V}$. These three quantities are considered together as they tend to be dominated by contributions from the low-latitude regions of the Sun. For the limited data simulations there is a strong variation in the agreement with the reference Sun simulation. While the agreement varies, it is always less than 1 , and the two limited data simulations tend to vary in phase with one another. It is clear that the Earth plus L5 simulation produces a much better agreement to the reference Sun simulation. Notice that all three quantities tend to be least accurate at cycle minimum. Again, this results from missing a proportion of the bipoles; the field at minimum is less dominated by a few active regions and therefore more sensitive to the cumulative properties of all bipoles over the cycle.

It is also useful to consider the average accuracy over the entire simulation through a calculation of average values of the quantities in Figure 10. These can be seen in Table 2, where the bottom row gives the percentage increase in accuracy obtained when L5 observations are included compared to the Earth-only 


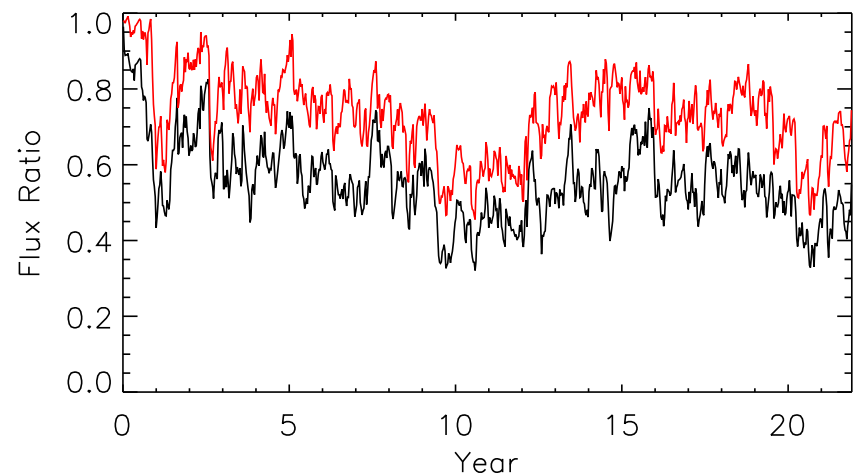

(a)

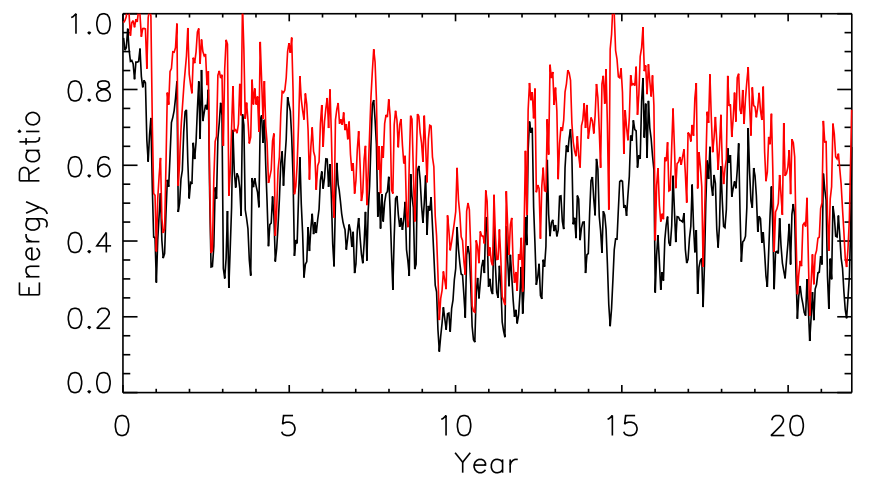

(b)

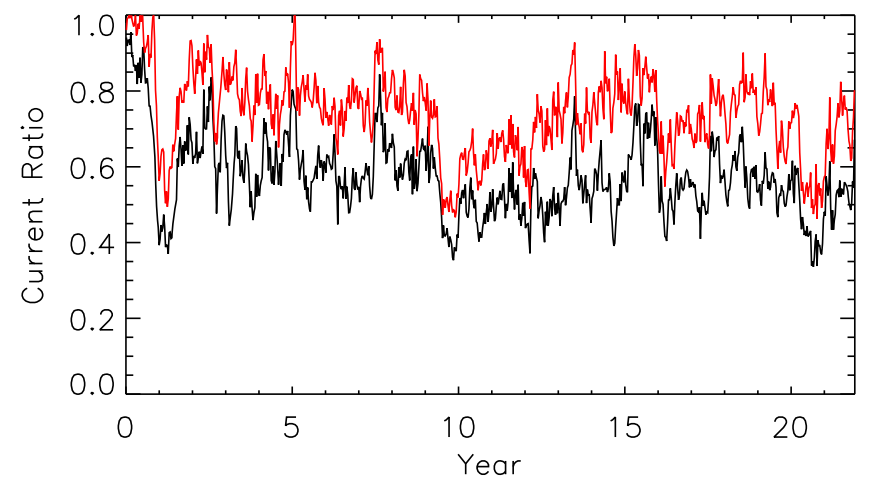

(c)

Figure 10. Comparison between the reference Sun simulation and the limited data simulations with the real-time emergence with rotational updates. In each case the black lines denote the Earth-only simulation and the red lines the Earth plus L5 simulation. Plotted quantities are given as a ratio with the reference Sun simulations and include (a) surface flux $\Phi_{s}$, (b) volume-integrated magnetic energy $E_{m}$, and (c) volume-integrated electric current $J_{V}$.

Table 2

Average Fit between the Limited Data Simulations and the Reference Sun Simulation for the Global Integrated Quantities

\begin{tabular}{lcccc}
\hline \hline Simulation & $\begin{array}{c}\text { Flux } \\
\text { Ratio }\end{array}$ & $\begin{array}{c}\text { Energy } \\
\text { Ratio }\end{array}$ & $\begin{array}{c}\text { Current } \\
\text { Ratio }\end{array}$ & $\begin{array}{c}\text { Flux } \\
\text { Ropes Ratio }\end{array}$ \\
\hline Earth only & $0.55(0.4)$ & $0.46(0.31)$ & $0.57(0.42)$ & $0.615(0.46)$ \\
Earth plus L5 & $0.75(0.56)$ & $0.65(0.465)$ & $0.74(0.58)$ & $0.78(0.6)$ \\
Improvement & $33 \%(40 \%)$ & $41 \%(50 \%)$ & $30 \%(38 \%)$ & $26 \%(30 \%)$ \\
\hline
\end{tabular}

Note. Values in parentheses are for simulations where only real-time emergences are used.

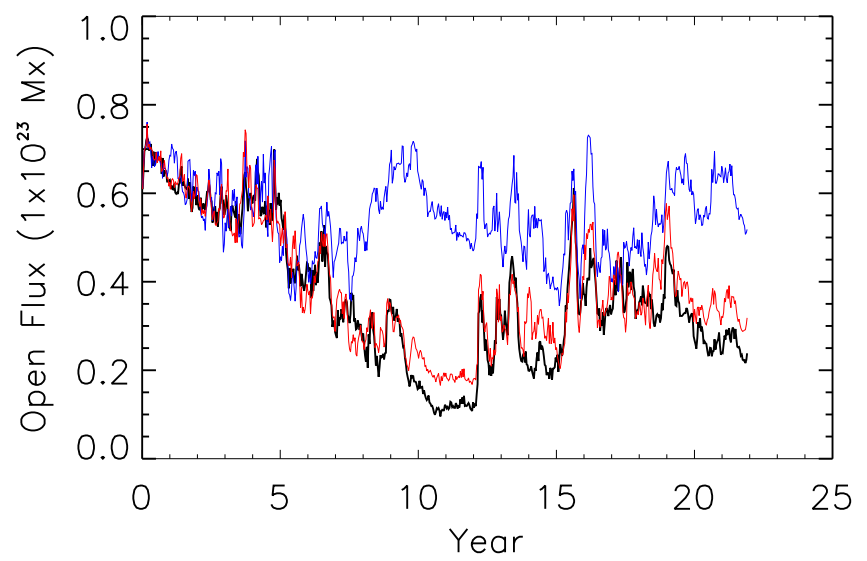

Figure 11. Open flux vs. time in the reference Sun simulation (blue line), Earth-only simulation (black line), and Earth plus L5 simulation (red line).

case. In this table the numbers given in parentheses are included for illustrative purposes and are for simulations where only real-time emergences are included.

From Table 2 it is clear that for the surface flux, magnetic energy, and electric current, anywhere from a $31 \%$ to $40 \%$ improvement can result from including L5 observations. Although a greater increase in accuracy occurs for the simulations where only real-time emergences are included, these simulations are less accurate overall. These increases show a significant increase in accuracy of the results through only including an extra $60^{\circ}$ in the FOV. Also of note is that the overall accuracy of these quantities in the Earth plus L5 case lies between $65 \%$ and $75 \%$, which is much better than the $46 \%-57 \%$ found in the Earth-only simulation.

In Figure 11 the variation of the open magnetic flux for the reference Sun and limited data simulations can be seen. In contrast to the quantities that are plotted in Figure 10, the actual values are shown rather than a ratio. This is so that the variations of the open flux can be understood. It should also be noted that sometimes more open flux is found in the limited data simulations, compared to the reference Sun simulation. Due to this, computing an average fit over the full simulation time period is not appropriate. The reason for this will be discussed in Section 5.2.1. From the graph it can be seen that both of the limited data simulations (black-Earth only; redEarth plus L5) follow the behavior of the reference Sun simulation (blue line) closely until around year 6 , where the curves diverge. After this, the limited data simulations give significantly less open flux at cycle minimum. While both cases produce less, the Earth plus L5 case gives a slightly better fit. Although there is a significant difference at cycle minimum, the differences decrease during the high-activity periods of the second cycle between years 15 and 19. This is when the open flux has a significant contribution from the low-latitude field regions.

As we have noted, the greatest disagreement between the limited data and reference Sun simulations occurs at cycle minimum because insufficient flux is pushed poleward in each cycle to first reverse the polar fields at the correct time and then build a polar field of the correct magnitude (compare Figure 11 with Figure 9). While quantities such as magnetic energy and electric current are insensitive to the amount of flux pushed 


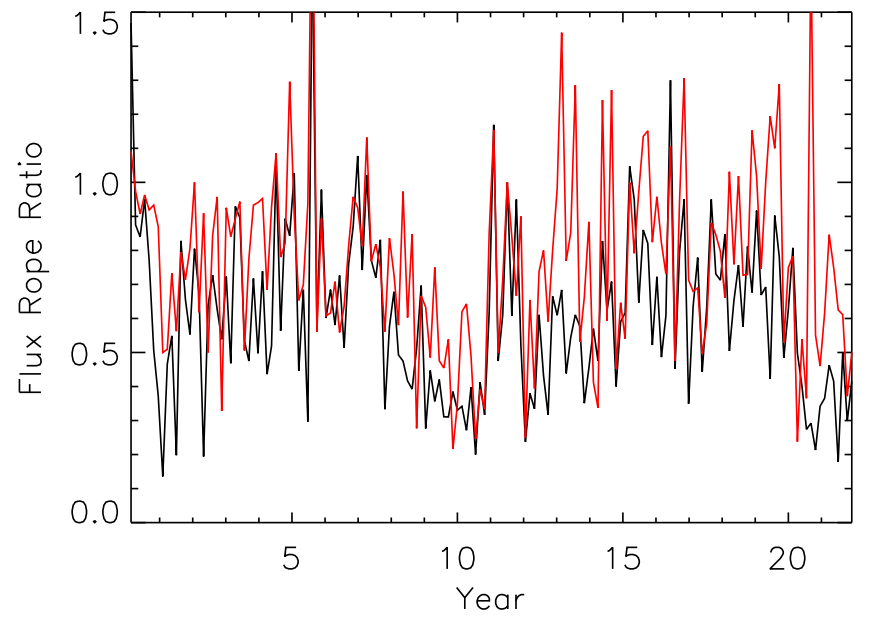

Figure 12. Comparison between the number of flux ropes in the reference Sun and limited data simulations, where real-time emergences with rotational updates are included. In each case the back lines denote the Earth-only simulation and the red lines the Earth plus L5 simulation. The plotted quantities are given as a ratio with the reference Sun simulations.

poleward (since they are dominated by low-latitude contributions), the behavior of the open flux and high-latitude regions is very sensitive to this. This is because the amount of flux held in the poles is comparable to just a few large magnetic bipoles. This shows that any model that tries to produce the highlatitude fields by emerging bipoles at low latitudes, when there is only a limited FOV to deduce these bipoles, will have difficulty in producing the correct polar field strength. This has very important implications for future operational space weather models. However, models that use observations to assimilate higher-latitude fields should be more accurate as they directly capture the missing flux that is transported poleward (although not at a single instant in time). One possible way to improve these results in the context of our global model is to not only emerge bipoles at low latitudes but also to correct the higher-latitude fields based on the amount of magnetic flux that is transported poleward. Techniques that can apply this using synoptic magnetograms such as ADAPT are currently under development (see Weinzierl et al. 2016). This will be discussed further in the conclusions.

\subsubsection{Number of Flux Ropes}

In Figure 12 a comparison is carried out between the number of flux ropes in the limited data simulations and that in the reference Sun simulation. Figure 12 shows the results for simulations where both real-time emergences and rotational updates are included. As in Figure 10, the red line shows the simulations where Earth plus L5 observations are used, and the black line is for Earth-only observations (again sampled once every 50 days). In the graph the ratio of the number of flux ropes in the limited data simulations to that of the reference Sun simulation (Figure 4(b)) is plotted. It is clear that when L5 observations are included, the limited data simulations reproduce much better the behavior of the reference Sun simulation (i.e., a value closer to 1). The overall accuracy of these simulations is given in the final column of Table 2. Through including L5 observations the accuracy improves by at least $26 \%$, capturing on average $78 \%$ of the number of flux ropes found in the reference Sun simulation. Since flux ropes are one of the mechanisms proposed to produce CMEs, the improvement in accuracy is an important factor that can arise if magnetograph data are included from the L5 point. For space weather forecasting this gives a significant improvement. One feature that requires discussion in Figure 12 is that the ratio may be greater than 1 for short periods of time. This means that at some instances there are more flux ropes present in the limited data simulations compared to the reference Sun simulation. To understand why this may arise, the nature of the formation and loss of flux ropes has to be considered. Within the simulations flux ropes may form at PILs where field lines are first sheared by differential rotation and then cancel. During the cancellation process, a longer field line is produced that lies above the PIL (see van Ballegooijen \& Martens 1989; Mackay \& van Ballegooijen 2006). As more and more flux cancels, the flux ropes grow and eventually become too large to be held down by their overlying arcades. Once this happens, the flux ropes are ejected from the simulation box and may produce CMEs (Pagano et al. 2014). Therefore, the number of flux ropes at any time varies, depending on (i) the number of locations where flux cancellation can take place, (ii) how fast it takes place, and finally (iii) the lifetime of the ropes. The latter property itself depends on the configuration and strength of the overlying field. Due to the fact that there are a number of competing processes affecting the number of flux ropes, it is not surprising that for limited time periods more flux ropes may exist in the limited data simulations compared to the reference Sun simulation. This is likely to be due to flux ropes that have already erupted in the reference Sun simulation but erupt at a later time in the limited data simulations. A later eruption occurs as the bipole is emerged at a later time and in a less stressed state. The flux ropes that erupt then result in an increase of open flux.

While the numbers in Table 2 show a significant improvement, it should be noted that in this comparison we are just comparing numbers of flux ropes. We are not comparing the accuracy in their spatial location or in the timing of when they become unstable and are ejected out of the top boundary. Due to the increased accuracy of the surface field in the Earth plus L5 simulation, it is expected that both the location of flux ropes and their timing of eruptions relative to the reference Sun simulation should be much more accurate compared to the Earth-only simulation. This should be especially true for those flux ropes existing at longitudes that are rotating toward $\mathrm{CM}_{\mathrm{E}}$ due to the earlier inclusion of the bipoles. In order to investigate this in detail, a 4D cross-correlation (space and time) of each of the limited data simulations with the reference Sun simulation will have to be carried out. Such a correlation is extremely time-consuming and is beyond the scope of the present paper, but will be considered in a follow-up study (see Section 6).

\section{DISCUSSION AND CONCLUSIONS}

In recent years, there has been significant interest in flying a satellite to the L5 point that lies $60^{\circ}$ behind Earth. This is to provide an extended view of the Sun and heliosphere for operational space weather forecasting. The payload of such a mission would consist of a variety of remote sensing and in situ instruments for observing the Sun's atmosphere and heliosphere. One possible instrument is an LOS magnetograph that will increase the longitudinal range of accurate magnetic field measurements at the photosphere by $60^{\circ}$, compared to what is currently possible. In the present paper, we have carried out a 
speculative study, to quantify what type of improvement could occur in global nonpotential modeling of the Sun's coronal magnetic field if an LOS magnetograph were to provide data from L5. Improved modeling of the Sun's nonpotential coronal magnetic field is necessary both to understand the origin of and in future to predict space weather sources on the Sun. For the present paper we focus on the global nonpotential model of Mackay \& van Ballegooijen (2006) and Yeates (2014), which uses well-observed large-scale motions and the emergence of isolated bipoles to drive the evolution of the coronal field. While we have focused on this technique, a wide variety of nonpotential models exist (see Mackay \& Yeates 2012), each with different requirements for producing the nonpotential fields. It is important that other authors carry out similar studies to quantify what improvements can occur in their models.

To determine what type of improvement can occur in our model, we have compared three nonpotential simulations where each simulation lasts for two solar cycles. The simulations extend over two solar cycles so that we can quantify the effect of an L5 magnetograph on both the low- and high-latitude fields. In the first simulation, which we name the "reference Sun" simulation, we assume that bipoles may emerge at any longitude on the Sun at any time. This represents the ideal case, which would arise if we had full coverage of the solar surface with magnetograph observations. Following this, two limited data simulations are carried out, termed "Earthonly" and "Earth plus L5." The Earth-only simulation assumes that we have magnetograph data available only from the SunEarth line and represents the current situation. In contrast, the Earth plus L5 case extends the magnetograph data by a further $60^{\circ}$. The accuracy of the two limited data simulations in reproducing the reference Sun simulation is then considered, along with the level of improvement that could occur if magnetograph data were available from the L5 point.

In the present paper, we have mainly focused on comparing global integrated quantities of the nonpotential surface and coronal fields. The first set of these quantities includes the surface flux, total magnetic energy, magnitude of the electric current, and the number of flux ropes. For each of these quantities the dominant part of the Sun that contributes to them is the low active latitude regions. Accurately reproducing these quantities is key for any future models that wish to predict where eruptive phenomena will occur on the Sun. Averaged over the two cycles, the Earth plus L5 simulation gives a 30\%$41 \%$ increase in accuracy for the first three quantities compared to the Earth-only simulation. The increase in accuracy for the number of flux ropes is slightly less at $26 \%$. The increased accuracy in the Earth plus L5 simulation is mostly due to its ability to capture the evolution of more of the magnetic bipoles. It therefore captures more fully their contribution to the complexity of both the surface and coronal fields and to the Poynting flux of energy that is injected into the corona.

While the global quantities, which are dominated by lowlatitude contributions, show a significant improvement, the open flux, which has a significant high-latitude contribution, shows only a small improvement. The reason for this can be understood by considering how flux is transported poleward in the simulation to produce the polar fields. From Figure 12 it is clear that neither of the limited data simulations captures the variation of the high-latitude polar fields correctly. In particular, the reversal occurs too late/early in the first/second cycle, along with the polar fields being too weak at the start of the second cycle. Notwithstanding these limitations, the Earth plus L5 case does produce the more accurate results for both the polar fields and open flux. In the simulations the polar fields at any given time are a consequence of the initial polar field strength and the amount of flux that is transported poleward. The initial polar field strength is the same for all of our simulations, but the latter quantity depends on the number of bipole emergences and bipole tilt angles, along with the rate and profile of meridional flow. While each bipole only contributes a small amount to the polar flux, the combined effect of missing $45.4 \%$ of the bipoles in the Earth-only simulation and $29.7 \%$ in the Earth plus L5 simulation is significant for both the open flux and polar fields. The sensitivity of the polar fields to even a single bipole has been discussed in the paper by Yeates et al. (2015). It should, however, be noted that while including L5 observations has only slightly increased the accuracy of the open flux, it is no worse than that which arises with our present Earth-only viewpoint. However, significant improvement is still found in the other quantities that are dominated by low-latitude contributions.

The results for the open flux described above illustrate a problem for any current or future model that wishes to continuously simulate the Sun's photospheric and coronal fields through the injection and transport of new magnetic bipoles at active latitudes. For these simulations the amount of flux pushed poleward very much depends on the number of bipoles that emerged and the bipole properties. Even if a small number of bipoles are missed due to fragmenting on the far side of the Sun, this can have a significant effect on the polar fields and open flux produced in the model. As a consequence, future models derived from observations must consider including not only both bipoles at active latitudes but potentially additional updates so as to accurately reproduce the amount of flux that is pushed poleward above $40^{\circ}$ latitude. It is critical to capture the net effect of decayed active regions and their contribution to the high-latitude transport of flux out of active regions. One way to do this is to assimilate magnetogram data over the entire visible disk rather than solely in the form of magnetic bipoles. Such an approach is used, for example, in the flux transport models of Schrijver \& DeRosa (2003) and Upton \& Hathaway (2014), as well as in the ADAPT model (Arge et al. 2010; Henney et al. 2012). Nonpotential coronal models using the ADAPT maps to produce time-evolving lower boundary conditions are currently under development (e.g., Weinzierl et al. 2016).

While placing a magnetograph at the L5 point is a direct method of increasing our observational range of photospheric magnetic fields and improving global magnetic field modeling, an alternative indirect method for deducing solar activity and active regions exists. This is based on inferred heliospheric farside detection of active regions (González Hernández et al. 2007). Currently this technique can be used to provide an estimate of the field strength and size of active regions on unobserved portions of the disk. While it can provide this information, for accurate nonpotential coronal field modeling, information on the spatial distribution of flux and the orientation of the polarities is required. Currently such information cannot be obtained (MacDonald et al. 2015); however, in the future, with further development, it may become possible to produce a powerful tool that can be combined with global modeling. While far-side imaging may be seen as an alternative to an L5 mission, both techniques 
complement one another. Currently it takes between 2 and 5 days of averaging signals to deduce new regions. The upper limit of this corresponds to the time frame where active regions may emerge in the L5 FOV and then rotate into the Earth FOV. Thus, such regions would not be detected in time using far-side imaging, but would be fully captured using an L5 magnetograph. In contrast, the present paper has also shown that missed active regions, outside the FOV of either Earth or L5, can seriously affect the open flux and polar fields. Liewer et al. (2014) have shown that over a 9-month period up to $50 \%$ of active regions emerging on the far side can be detected using far-side imaging. Thus, the use of combined Earth, L5, and farside imaging of active regions (assuming that detailed magnetic information is obtained) could significantly reduce the number of missed regions and, with this, increase the accuracy of the high-latitude global fields.

While the present study shows some interesting results in terms of the improvement that an L5 magnetograph can have on global nonpotential simulations, currently only the simplest of comparisons has taken place. In a follow-up paper we will consider a much more detailed analysis of the local properties of the limited data simulations with the reference Sun simulation. In particular, we will carry out a 4D correlation analysis (space and time) for (i) the location and size of free magnetic energy storage, (ii) the location and size of flux ropes, and finally (iii) the timing and properties of erupting flux ropes. An important part of this study will be considering the latter quantity for flux ropes within $\pm 60^{\circ}$ of the Earth-based central meridian, which are important for future space weather predictions. A local rather than global analysis will allow a much more detailed understanding of the accuracy of the limited data simulations. While such a study is extremely important, its time-consuming nature puts it beyond the scope of the present paper.

As well as carrying out the above comparison, it will also be useful to compare the reference Sun simulation with synoptic magnetograms derived from the limited data simulations. The synthetic synoptic magnetograms can be constructed from both the Earth and Earth plus L5 viewpoints. This will produce a closer comparison to what can occur in reality compared to the comparisons carried out in the present paper. It will be interesting to determine whether such a comparison can produce a much more accurate representation of the polar fields and open flux compared to the present simulation technique.

Notwithstanding these possible future studies, the present study illustrates that an L5 mission could significantly increase the accuracy of global nonpotential models. This is particularly true in the complex energy storage regions around the active region belt of the Sun.

D.H.M. would like to thank STFC and the Leverhulme Trust for their financial support. A.R.Y. would like to thank STFC and the U.S. Air Force for financial support. Both authors participated in a team investigation on global magnetic fields at the International Space Science Institute and are grateful to ISSI for hosting us.

\section{APPENDIX}

In order to include the bipoles that emerge late, with updated magnetic flux due to their prior evolution, we develop a new analytic model that describes the evolution of a bipolar magnetic region under the effects of advection and surface diffusion. We solve the evolution of the bipolar magnetic region analytically to obtain a closed-form solution (see Leighton 1964; DeVore et al. 1984; DeVore 1987). We choose to consider a local solution in planar geometry, as we are only considering the evolution of a single bipolar active region over a short period of time. Under this approximation, the equation of surface flux transport becomes

$$
\begin{aligned}
\frac{\partial B_{z}}{\partial t}= & -v_{x} \frac{\partial B_{z}}{\partial x}-v_{y} \frac{\partial B_{z}}{\partial y} \\
& +D\left(\frac{\partial^{2} B_{z}}{\partial x^{2}}+\frac{\partial^{2} B_{z}}{\partial y^{2}}\right)
\end{aligned}
$$

where $D$ is the rate of surface diffusion, the $(x, y)$ plane represents the photosphere, and $\nabla . \boldsymbol{v}=0$ (DeVore et al. 1984; DeVore 1987). The $x$ direction lies in the direction of $\phi$ and $y$ in $\theta$. Here $v_{x}(x, y)$ represents differential rotation and $v_{y}(x, y)$ meridional flow. To allow an analytic solution, we choose the incompressible steady flow,

$$
\begin{gathered}
v_{x}=-\Omega_{o} y, \\
v_{y}=u_{o},
\end{gathered}
$$

which is a good approximation to the real profiles found on the Sun over a localized region. We solve the flux transport equation in a Lagrangian coordinate system, where the trajectory of a fluid element initially at the location $\boldsymbol{x}=\boldsymbol{a}$ is given by

$$
\frac{\partial \boldsymbol{x}}{\partial t}=\boldsymbol{v}(\boldsymbol{x}, t), \quad \boldsymbol{x}(t=0)=\boldsymbol{a} .
$$

Using the velocity profiles given in Equations (15) and (16), the transformation between the Eulerian $(\boldsymbol{x})$ and Lagrangian $(\boldsymbol{a})$ coordinates is

$$
\begin{gathered}
y(t ; \boldsymbol{a})=u_{o} t+a_{2} \\
x(t ; \boldsymbol{a})=-\frac{1}{2} \Omega_{o} u_{o} t^{2}-\Omega_{o} t a_{2}+a_{1}
\end{gathered}
$$

therefore,

$$
\begin{gathered}
a_{1}=x-\frac{1}{2} \Omega_{o} u_{o} t^{2}+\Omega_{o} t y, \\
a_{2}=y-u_{o} t
\end{gathered}
$$

In these coordinates, the flux transport equation becomes an anistropic diffusion equation,

$$
\begin{aligned}
\frac{\partial B_{z}}{\partial t}= & D\left(1+\Omega_{o}^{2} t^{2}\right) \frac{\partial^{2} B_{z}}{\partial a_{1}^{2}} \\
& +2 D \Omega_{o} t \frac{\partial^{2} B_{z}}{\partial a_{1} \partial a_{2}}+D \frac{\partial^{2} B_{z}}{\partial a_{2}^{2}} .
\end{aligned}
$$

At $t=0$ this reduces to the standard diffusion equation, but at later times the diffusion is enhanced due to the deformation of $B_{z}$ by advection. We may solve Equation (22) by taking a 2D Fourier transform in $\boldsymbol{a}$, where we define

$$
\begin{aligned}
& B_{z}(\boldsymbol{a}, t) \\
& \quad=\int_{-\infty}^{\infty} \int_{-\infty}^{\infty} \hat{B}_{z}(\boldsymbol{k}, t) e^{i k_{1} a_{1}+i k_{2} a_{2}} d k_{1} d k_{2},
\end{aligned}
$$




$$
\begin{aligned}
& \hat{B}_{z}(\boldsymbol{k}, t) \\
& \quad=\frac{1}{(2 \pi)^{2}} \int_{-\infty}^{\infty} \int_{-\infty}^{\infty} B_{z}(\boldsymbol{a}, t) e^{-i k_{1} a_{1}-i k_{2} a_{2}} d a_{1} d a_{2} .
\end{aligned}
$$

After substituting Equation (23) into Equation (22), we obtain the time evolution of the Fourier transform $\hat{B}_{z}$,

$$
\begin{aligned}
& \frac{1}{D} \frac{\partial \hat{B}_{z}}{\partial t} \\
& \quad=-\left\{k_{1}^{2}\left(1+\Omega_{o}^{2} t^{2}\right)+2 \Omega_{o} t k_{1} k_{2}+k_{2}^{2}\right\} \hat{B}_{z},
\end{aligned}
$$

whose solution is

$$
\begin{aligned}
& \hat{B}_{z}(\boldsymbol{k}, t) \\
& \quad=\hat{A}(\boldsymbol{k}) e^{\left\{-D\left(k_{1}^{2} t+k_{2}^{2} t+\Omega_{o} k_{1} k_{2} t^{2}+\frac{1}{3} \Omega_{o}^{2} k_{1}^{2} t^{3}\right)\right\}},
\end{aligned}
$$

where $\hat{A}(\boldsymbol{k})$ is the Fourier transform of the initial condition $B_{z}(\boldsymbol{a}, t=0)$. Once $\hat{B}_{z}(\boldsymbol{k}, t)$ is known, we may invert the transform using Equation (23), where Equations (20) and (21) can be used to find the solution in terms of $(x, y)$.

If we now consider an initial condition of a bipole inserted into the simulation with a half separation of $\rho_{o}$ and tilt angle $\gamma_{o}$ where

$$
B_{z}(x, y, 0)=-B_{o} e^{1 / 2} \frac{x^{\prime}}{\rho_{o}} e^{-\xi}, \quad \xi=\frac{\left(x^{\prime}\right)^{2} / 2+y^{\prime 2}}{\rho_{o}^{2}},
$$

and

$$
\begin{aligned}
& x^{\prime}=\left(x-x_{o}\right) \cos \gamma_{o}-\left(y-y_{0}\right) \sin \gamma_{o}, \\
& y^{\prime}=\left(x-x_{o}\right) \sin \gamma_{o}+\left(y-y_{0}\right) \cos \gamma_{o},
\end{aligned}
$$

then since the Eulerian and Lagrangian coordinates match at $t=0$,

$$
B_{z}(\boldsymbol{a}, 0)=-B_{o} e^{1 / 2} \frac{a_{1}^{\prime}}{\rho_{o}} e^{-\xi}, \quad \xi=\frac{\left(a_{1}^{\prime}\right)^{2} / 2+a_{2}^{\prime 2}}{\rho_{o}^{2}},
$$

with

$$
\begin{aligned}
& a_{1}^{\prime}=\left(a_{1}-x_{o}\right) \cos \gamma_{o}-\left(a_{2}-y_{0}\right) \sin \gamma_{o}, \\
& a_{2}^{\prime}=\left(a_{1}-x_{o}\right) \sin \gamma_{o}+\left(a_{2}-y_{0}\right) \cos \gamma_{o} .
\end{aligned}
$$

The solution for this initial condition may be obtained in closed form from Equations (23) and (26), giving

$$
\begin{aligned}
B_{z}(\boldsymbol{a}, t)= & \frac{-B_{o} \rho_{o}^{3} \sqrt{2 e}}{\left(4 A C-B^{2}\right)^{3 / 2}} \\
& \times\left(\sin \gamma_{o}\left[\frac{1}{2} B \bar{a}_{1}-A \bar{a}_{2}\right]\right. \\
& -\left(\cos \gamma_{o}\left[\frac{1}{2} B \bar{a}_{2}-C \bar{a}_{1}\right]\right) \\
& \times \exp \left\{\frac{-C \bar{a}_{1}^{2}+B \bar{a}_{1} \bar{a}_{2}-A \bar{a}_{2}^{2}}{4 A C-B^{2}}\right\}
\end{aligned}
$$

where $\bar{a}_{1}=a_{1}-x_{o}, \bar{a}_{2}=a_{2}-y_{o}$, and

$$
\begin{aligned}
A(t)= & \frac{1}{4} \rho_{o}^{2} \sin ^{2} \gamma_{o}+\frac{1}{2} \rho_{o}^{2} \cos ^{2} \gamma_{o} \\
& +D t+\frac{1}{3} D \Omega_{o}^{2} t^{3},
\end{aligned}
$$

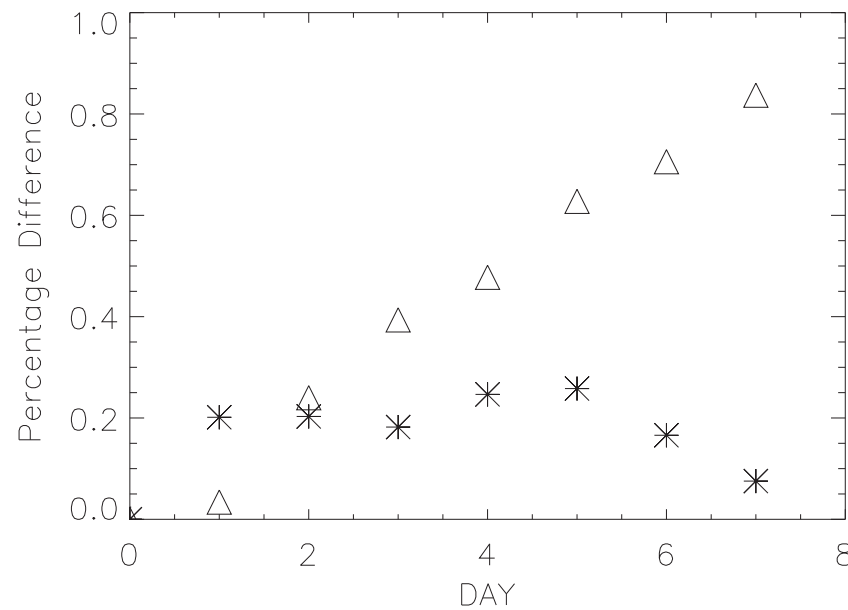

Figure 13. Percentage difference between the simulated flux and analytical model flux over a period of 7 days. The stars denote a bipole placed at latitude $10^{\circ}$, while the triangles denote a bipole at $40^{\circ}$.

$$
\begin{gathered}
B(t)=-\frac{1}{2} \rho_{o}^{2} \sin \gamma_{o} \cos \gamma_{o}+D \Omega_{o} t^{2}, \\
C(t)=\frac{1}{4} \rho_{o}^{2} \cos ^{2} \gamma_{o}+\frac{1}{2} \rho_{o}^{2} \sin ^{2} \gamma_{o}+D t,
\end{gathered}
$$

where these terms include the time dependence. Care must be taken as the solution involves the factor $Q(t)=\left(4 A C-B^{2}\right)$, where a valid solution is only found for $Q \neq 0$ and $Q^{3 / 2}$ real. For all of the bipole tilt angles considered here and evolution over short time periods, this is found to be true. After integrating over all space and further significant manipulation, the variation of the total magnetic flux is

$$
\Phi(t)=B_{o} \rho_{o}^{3} \sqrt{2 \pi e} \sqrt{\frac{W(t)}{Q(t)}}
$$

where

$$
W(t)=A \sin ^{2} \gamma_{o}+B \sin \gamma_{o} \cos \gamma_{o}+C \cos ^{2} \gamma_{o}
$$

Note that at $t=0, \Phi(0)=B_{o} \rho_{o}^{2} \sqrt{e \pi}$, which is the correct value. Using Equation (37), we may determine the change in flux over a given time period.

To illustrate the validity of the technique where we use a closed-form analytic solution in Cartesian geometry, we carry out a comparison between the analytic model and a numerical solution in spherical geometry. This can be seen in Figure 13, where we see a graph of the percentage difference between the two modeling approaches as a function of time. In each case a bipole with tilt angle of $10^{\circ}$ is simulated from an initial latitude of either $10^{\circ}$ (stars) or $40^{\circ}$ (triangles). From the graph it is clear that there is only a minor difference between the two results, with the difference less than $1 \%$. It is also clear from the graph that the error is much less for the bipole located at a latitude of $10^{\circ}$. This is due to the differential rotation profile of the Sun being closer to linear at lower latitudes. While the error is small over the time period considered, for the higher-latitude bipole the error does increase sharply. Therefore, care would have to be taken to extend the use of the analytic model beyond the 7 days used in the present study. 


\section{REFERENCES}

Arge, C. N., Henney, C. J., Koller, J., et al. 2010, in AIP Conf. Proc. 1216 , Twelfth Int. Solar Wind Conf. 1216, ed. M. Maksimovic et al. (Melville, NY: AIP), 343

Balogh, A., Smith, E. J., Tsurutani, B. T., et al. 1995, Sci, 268, 1007

Benz, A. O. 2008, LRSP, 5, 1

Bhattacharjee, A., \& Hameiri, E. 1986, PhRvL, 57, 206

Boozer, A. H. 1986, JPIPh, 35, 133

Contopoulos, I., Kalapotharakos, C., \& Georgoulis, M. K. 2011, SoPh, 269, 351

DeVore, C. R. 1987, SoPh, 112, 17

DeVore, C. R., \& Antiochos, S. K. 2008, ApJ, 680, 740

DeVore, C. R., Boris, J. P., \& Sheeley, N. R., Jr. 1984, SoPh, 92, 1

DeVore, C. R., Sheeley, N. R., Jr., Boris, J. P., Young, T. R., Jr., \& Harvey, K. L. 1985, SoPh, 102, 41

Downs, C., Roussev, I. I., van der Holst, B., et al. 2010, ApJ, 712, 1219

Duvall, T. L., Jr. 1979, SoPh, 63, 3

Feng, X., Yang, L., Xiang, C., et al. 2012, SoPh, 279, 207

Fontenla, J. M., Curdt, W., Haberreiter, M., Harder, J., \& Tian, H. 2009a, ApJ, 707,482

Fontenla, J. M., Quémerais, E., González Hernández, I., Lindsey, C., \& Haberreiter 2009b, AdSpR, 44, 457

Forbes, T. G., Linker, J. A., Chen, J., et al. 2006, SSRv, 123, 251

González Hernández, I., Hill, F., \& Lindsey, C. 2007, ApJ, 669, 1382

Harvey, K. L., \& Zwaan, C. 1993, SoPh, 148, 85

Henney, C. J., Hock, R. A., Schooley, A. K., et al. 2015, SpWea, 13, 141

Henney, C. J., Toussaint, W. A., White, S. M., \& Arge, C. N. 2012, SpWea, $10, \mathrm{~S} 02011$

Hollweg, J. V. 2008, JApA, 29, 217

Labrosse, N., Heinzel, P., Vial, J.-C., et al. 2010, SSRv, 151, 243

Leighton, R. B. 1964, ApJ, 140, 1547

Liewer, P. C., González Hernández, I., Hall, J. R., Lindsey, C., \& Lin, X. 2014, SoPh, 289, 3617

Lionello, R., Linker, J. A., \& Mikić, Z. 2009, ApJ, 690, 902

Lockwood, M., Forsyth, R., Balogh, A., \& McComas, D. 2004, AnGeo, 22, 1395
MacDonald, G. A., Henney, C. J., Díaz Alfaro, M., et al. 2015, ApJ, 807, 21 Mackay, D., \& Yeates, A. 2012, LRSP, 9, 6

Mackay, D. H., Karpen, J. T., Ballester, J. L., Schmieder, B., \& Aulanier, G. 2010, SSRv, 151, 333

Mackay, D. H., \& van Ballegooijen, A. A. 2006, ApJ, 641, 577

Odstrcil, D., \& Pizzo, V. 1999, JGR, 104, 483

Pagano, P., Mackay, D. H., \& Poedts, S. 2013, A\&A, 560, A38

Pagano, P., Mackay, D. H., \& Poedts, S. 2014, A\&A, 568, A120

Riley, P., Linker, J. A., Mikić, Z., et al. 2006, ApJ, 653, 1510

Schrijver, C. J., \& DeRosa, M. L. 2003, SoPh, 212, 165

Schrijver, C. J., \& Harvey, K. L. 1994, SoPh, 150, 1

Schüssler, M., \& Baumann, I. 2006, A\&A, 459, 945

Sheeley, N. R., Jr. 2005, LRSP, 2, 5

Snodgrass, H. B. 1983, ApJ, 270, 288

Tian, L., Zhang, H., Tong, Y., \& Jing, H. 1999, SoPh, 189, 305

Upton, L., \& Hathaway, D. H. 2014, ApJ, 780, 5

van Ballegooijen, A. A., Cartledge, N. P., \& Priest, E. R. 1998, ApJ, 501, 866

van Ballegooijen, A. A., \& Cranmer, S. R. 2008, ApJ, 682, 644

van Ballegooijen, A. A., \& Martens, P. C. H. 1989, ApJ, 343, 971

van Ballegooijen, A. A., Priest, E. R., \& Mackay, D. H. 2000, ApJ, 539, 983

Wang, Y.-M., \& Sheeley, N. R., Jr. 1989, SoPh, 124, 81

Weinzierl, M., Yeates, A. R., Mackay, D. H., Henney, C. J., \& Arge, C. N. 2016, ApJ, 823, 55

Wiegelmann, T. 2007, SoPh, 240, 227

Wiegelmann, T., Neukirch, T., Ruan, P., \& Inhester, B. 2007, A\&A, 475, 701

Worden, J., \& Harvey, J. 2000, SoPh, 195, 247

Yang, W. H., Sturrock, P. A., \& Antiochos, S. K. 1986, ApJ, 309, 383

Yeates, A. R. 2009, PhD thesis, Univ. St Andrews

Yeates, A. R. 2014, SoPh, 289, 631

Yeates, A. R., Baker, D., \& van Driel-Gesztelyi, L. 2015, SoPh, 290, 3189

Yeates, A. R., \& Mackay, D. H. 2009, ApJ, 699, 1024

Yeates, A. R., \& Mackay, D. H. 2012, ApJL, 753, L34

Yeates, A. R., Mackay, D. H., \& van Ballegooijen, A. A. 2007, SoPh, 245, 87

Yeates, A. R., Mackay, D. H., \& van Ballegooijen, A. A. 2008, SoPh, 247, 103

Yeates, A. R., Mackay, D. H., van Ballegooijen, A. A., \& Constable, J. A. 2010, JGRA, 115, A09112 